\title{
Joint Spectrum Sensing and Access Evolutionary Game in Cognitive Radio Networks
}

\author{
Chunxiao Jiang, Member, IEEE, Yan Chen, Member, IEEE, Yang Gao, Student Member, IEEE, \\ and K. J. Ray Liu, Fellow, IEEE
}

\begin{abstract}
Many spectrum sensing methods and dynamic access algorithms have been proposed to improve the secondary users' opportunities of utilizing the primary users' spectrum resources. However, few of them have considered to integrate the design of spectrum sensing and access algorithms together by taking into account the mutual influence between them. In this paper, we propose to jointly analyze the spectrum sensing and access problem by studying two scenarios: synchronous scenario where the primary network is slotted and non-slotted asynchronous scenario. Due to selfish nature, secondary users tend to act selfishly to access the channel without contribution to the spectrum sensing. Moreover, they may take out-of-equilibrium strategies because of the uncertainty of others' strategies. To model the complicated interactions among secondary users, we formulate the joint spectrum sensing and access problem as an evolutionary game and derive the evolutionarily stable strategy (ESS) that no one will deviate from. Furthermore, we design a distributed learning algorithm for the secondary users to converge to the ESS. With the proposed algorithm, each secondary user senses and accesses the primary channel with the probabilities learned purely from its own past utility history, and finally achieves the desired ESS. Simulation results shows that our system can quickly converge to the ESS and such an ESS is robust to the sudden unfavorable deviations of the selfish secondary users.
\end{abstract}

Index Terms-Cognitive radio, joint spectrum sensing and access, evolutionary game theory, replicator dynamics.

\section{INTRODUCTION}

$\mathbf{R}$ ECENTLY, cognitive radio has been proposed as an effective communication paradigm to mitigate the problem of crowded radio spectrums. Through dynamic spectrum access (DSA), the utilization efficiency of existing spectrum resources can be greatly improved [1][2]. In DSA, cognitive devices, called as Secondary Users (SUs) can dynamically access the licensed spectrum in an opportunistic way, under the condition that the interference to the Primary Users (PUs) in the licensed spectrum is minimized [3].

To detect available spectrums, the SUs need to perform spectrum sensing to monitor the PUs' activities. Many spectrum sensing algorithms have been proposed in the literatures

Manuscript received August 3, 2012; revised November 19, 2012; accepted February 8,2013 . The associate editor coordinating the review of this paper and approving it for publication was S. Bahk.

C. Jiang is with the Department of Electrical and Computer Engineering, University of Maryland, College Park, MD 20742, USA, and also with with the Department of Electronic Engineering, Tsinghua University, Beijing 100084, P. R. China (e-mail:jcx@umd.edu).

Y. Chen, Y. Gao, and K. J. Ray Liu are with the Department of Electrical and Computer Engineering, University of Maryland, College Park, MD 20742, USA (e-mail: \{yan, yanggao, @umd.edu, kjrliu\}@umd.edu).

Digital Object Identifier 10.1109/TWC.2013.031813.121135
[3]-[8]. Spectrum sensing methods based on energy detection and waveform sensing were proposed in [4] and [5], respectively. To improve the sensing performance, Ghasemi et al. proposed cooperative spectrum sensing to combat shadowing/fading effects [6], while Visotsky et al. studied how to combine spectrum sensing results in cooperative spectrum sensing schemes [7]. In [8], the authors derived the closedform expressions for the detection and false-alarm probabilities of cooperative spectrum sensing. Two MAC-layer sensing modes were investigated in [9], including slotted-time sensing and continuous-time sensing. The distributed compressive spectrum sensing in cooperative multi-hop cognitive networks was studied in [10].

After detecting available spectrums, the SUs need to decide how to access the spectrum. Several spectrum access methods based on different mathematical models have been proposed, e.g., Markov decision process (MDP) based approaches [11], queuing theoretic approaches [12], and game theoretic approaches [13]. In [11], Zhao et al. proposed a decentralized spectrum access protocol based on partially observable MDP (POMDP). Later, the authors in [14] extended the POMDP framework with learning the unknown statistics of the primary signals. In [12][15][16], the SUs were modeled as separate queuing systems and a multiple-access strategy with cooperative relays was proposed. Wu et al. in [17] proposed a multi-winner spectrum auction game to suppress the SUs' collision behaviors during spectrum access, while Li et al. in [18] presented a coalitional game theoretic approach for the SUs' spectrum access.

However, most existing works separated the analysis of spectrum sensing and access, i.e., either optimizing the spectrum sensing performance without considering the effect of spectrum sharing, or designing the multi-user access algorithm without considering the issue of spectrum sensing. In this paper, we will integrate the analysis of the SUs' spectrum sensing and access by considering a joint spectrum sensing and access game. On one hand, when only a few SUs contribute to spectrum sensing, the false-alarm probability is relatively high, resulting in low throughput during channel access. On the other hand, when many SUs access the primary channel, the channel will be very crowded and little throughput can be obtained by an individual SU. Therefore, each SU should dynamically adjust its strategy accordingly through learning from its strategic interactions with other SUs. In [19], a joint design of spectrum sensing and access was studied from a queuing theoretic view, which considered the effect 
of spectrum sensing errors on the performance of the SUs' multiple channel access. In this paper, we propose a game theoretic framework for joint spectrum sensing and access by considering the mutual influence between spectrum sensing and access. In [20], a coalition game theoretic approach for joint spectrum sensing and access problem was proposed, which focused on how to form coalitions among the SUs to constitute a Nash-stable network partition. On the other hand, our evolutionary game approach in this paper is to derive evolutionarily stable strategies for the SUs.

Since the SUs are naturally selfish, they want to access the channel without contributing to the spectrum sensing. Moreover, they may take out-of-equilibrium strategies due to the uncertainty of others' strategies. Therefore, a robust Nash equilibrium (NE) is desired for each SU. To model these complicated interactions among the SUs and find a stable NE for them, we formulate the joint spectrum sensing and access problem as an evolutionary game and derive the evolutionarily stable strategy (ESS). Evolutionary game has been used to model users' behaviors in image processing [21], as well as communication and networking [22][23], such as congestion control [24], cooperative sensing [25], and cooperative peerto-peer (P2P) streaming [26]. In these literatures, evolutionary game has been demonstrated as an effective approach to model the dynamic social interactions among users in a network.

According to the PU's communication mechanism, we study the joint spectrum sensing and access evolutionary game under two scenarios. In the first scenario, the primary network is slotted and the SUs are synchronous with the PU, while in the second scenario, the primary network is not slotted and the SUs cannot be synchronous with the PU. We derive the ESSs of these two scenarios and discuss how to achieve a desired ESS distributively. In a distributed network, it is generally difficult for a SU to know others' strategies and the corresponding utilities. Moreover, the behaviors of the SUs are highly dynamic, i.e., they may join or leave the secondary network at any time. Considering those problems, we propose a distributed learning algorithm for the SUs to achieve the ESS purely based on their own utility histories.

The rest of this paper is organized as follows. Firstly, our system model is described in details in Section II. Then, we analyze the joint spectrum sensing and access problem using evolutionary game theory under synchronous and asynchronous scenarios in Section III and Section IV, respectively. In Section V, we present our proposed distributed learning algorithm. Finally, simulation results are shown in Section VI and conclusions are drawn in Section VII.

\section{SySTEM MODEL}

\section{A. Network Entity}

As shown in Fig. 1, we consider a cognitive radio network with one licensed primary channel and $M$ SUs. The PU has priority to access the channel at any time, while the SUs are allowed to temporarily occupy the channel under the condition that the PU's communication quality of service (QoS) is guaranteed. All SUs can independently perform spectrum sensing using energy detection and then report their sensing results to others. There is a narrow-band signalling channel in

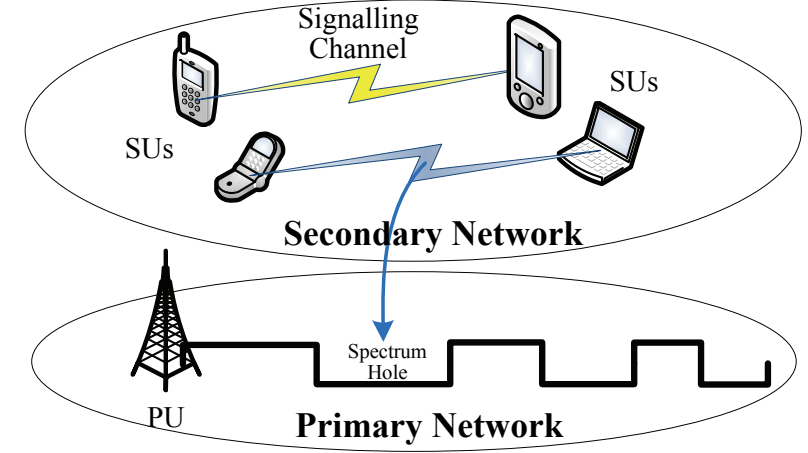

Fig. 1. Network entity.

the secondary network for the SUs to exchange their sensing results [27]. Nevertheless, the SUs need to opportunistically access the primary channel to acquire more bandwidth for high data rate transmission, e.g., multimedia transmission. Considering that the SUs are usually small-size and power-limit mobile terminals, we assume they are all half-duplex, which not only means that the SUs cannot simultaneously transmit and receive data, but also specially refers that they cannot perform spectrum sensing while keeping data communication [28].

\section{B. Spectrum Sensing Model}

Cooperative spectrum sensing technology can effectively overcome the channel fading and shadowing problems in the spectrum sensing [6]. In this paper, we adopt the distributed cooperative sensing architecture, in which each SU independently decides whether the PU is present through combining its own sensing results and other SUs'. To achieve better sensing performance, we assume SUs report their full sensing information to others, which is known as the soft combination rules [29].

Let $\mathcal{H}_{0}$ and $\mathcal{H}_{1}$ denote the PU being absent and present, respectively. The received signals by the SUs under $\mathcal{H}_{0}$ and $\mathcal{H}_{1}$ can be written by

$$
\begin{aligned}
& \mathcal{H}_{0}: y_{m}[n]=u_{m}[n], \\
& \mathcal{H}_{1}: y_{m}[n]=s_{m}[n]+u_{m}[n], \quad \text { the PU is present, }
\end{aligned}
$$

where $y_{m}[n]$ is $n$th sample of the $m$ th SU's sensing signal, $u_{m}$ is the noise, which is assumed to be Circular Symmetric Complex Gaussian (CSCG) with zero mean and variance $\sigma^{2}$ [8], and $s_{m}$ is the PU's signal, which is considered as an i.i.d random process with zero mean and variance $\sigma_{p}^{2}$. In such a case, for the $m$ th SU, the average energy of sensed signal $Y_{m}$ can be calculated by

$$
Y_{m}=\frac{1}{\lambda T_{s}} \sum_{n=1}^{\lambda T_{s}}\left|y_{m}^{(i)}[n]\right|^{2},
$$

where $T_{s}$ is the sensing time and $\lambda$ is the sampling rate. Then, all SUs send their own sensing data $Y_{m}$ to the whole network. Thus, the overall average energy $Y$ is

$$
Y=\frac{1}{M} \sum_{m=1}^{M} Y_{m}=\frac{1}{\lambda M T_{s}} \sum_{m=1}^{M} \sum_{n=1}^{\lambda T_{s}}\left|y_{m}[n]\right|^{2} .
$$




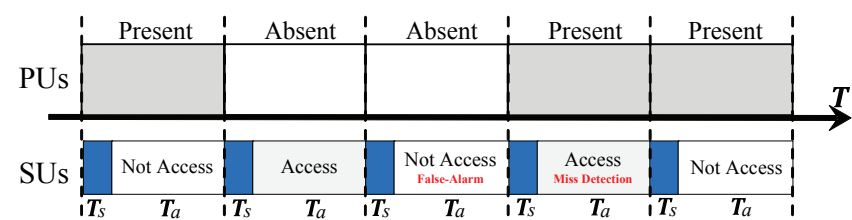

(a) Synchronous scenario.

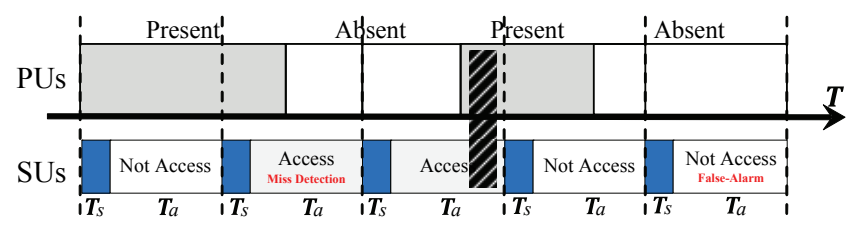

(b) Asynchronous scenario.

Fig. 2. Illustration of two scenarios.

Finally, the SUs compare $Y$ with a predetermined threshold $Y_{t h}$ to determine whether the $\mathrm{PU}$ is absent or present as follows:

$\mathcal{D}_{0}: \quad Y<Y_{t h}$, the SUs decide that the PU is absent,

$\mathcal{D}_{1}: \quad Y \geq Y_{t h}, \quad$ the SUs decide that the PU is present.

The performance of spectrum sensing is generally measured by two terms: detection probability $P_{d}$ and false-alarm probability $P_{f}$. The $P_{d}$ is defined as the probability that the SUs can detect the PU when the PU is present, while $P_{f}$ represents the probability that the SUs falsely report that the $\mathrm{PU}$ is present when the PU is actually absent. Since $P_{d}$ is usually pre-required by the $\mathrm{PU}$ in advance, the corresponding $P_{f}$ can be calculated by [25]

$$
\begin{aligned}
P_{f} & =\frac{1}{2} \operatorname{erfc}\left(\sqrt{2 \gamma+1} \cdot \operatorname{erfc}^{-1}\left(2 P_{d}\right)+\gamma \sqrt{\frac{M \lambda T_{s}}{2}}\right), \\
& =\frac{1}{2} \operatorname{erfc}\left(\Omega+\gamma \sqrt{\frac{M \lambda T_{s}}{2}}\right),
\end{aligned}
$$

where $\gamma=\sigma_{p} / \sigma$ is the SUs' received SNR of the PU's signal under $\mathcal{H}_{1}, \operatorname{erfc}(\cdot)$ is the complementary error function $\operatorname{erfc}(x)=\frac{2}{\sqrt{\pi}} \int_{x}^{+\infty} e^{-t^{2}} d t$.

\section{Synchronous and Asynchronous Scenarios}

In this paper, we will study the joint spectrum sensing and access problem under two scenarios: synchronous scenario and asynchronous scenario. In the synchronous scenario, we assume that the primary channel is slotted and the SUs are synchronous with the PU's time slots, as shown in Fig. 2-(a). In the asynchronous scenario, we assume that the primary communication is private and the SUs have no knowledge about the PU's exact communication behavior. In such a case, as shown in Fig. 2-(b), the system clock of the secondary network is asynchronous with that of the primary network [30]. For both scenarios, at the beginning of each slot, the SUs sense the primary channel with time $T_{s}$ and consider to access the channel with time $T_{a}$ if they observe that the PU is absent.

In the secondary network, each SU may try to maximize its access time to obtain most data throughput with least contributions to the spectrum sensing. The SUs' such selfish behavior will inevitably lead to the worst case that no one senses the primary channel, resulting in an extremely high false-alarm probability $P_{f}$ and thus nearly zero throughput. Moreover, even if all SUs contribute to the spectrum sensing, too many SUs accessing the primary channel will make the channel very crowded and again lead to very low throughput. In such a case, how should selfish but rational SUs collaborate with each others in spectrum sensing and access? Which SUs should perform sensing and which SUs should access the channel? In the following sections, we will answer the questions using evolutionary game for the synchronous and asynchronous scenarios, respectively.

\section{EVOLUTIONARY GAME FORMULATION FOR SYNCHRONOUS SCENARIO}

In this section, we first introduce the concept of evolutionary game, and then define the SUs' utility functions of joint spectrum sensing and access under the synchronous scenario. Through analyzing the replicator dynamics equations, we derive the SUs' evolutionarily stable strategy (ESS).

\section{A. Evolutionary Game}

1) Evolutionarily Stable Strategy: In the evolutionary game, each player dynamically adjusts his/her strategy through observing the utilities under different strategies. It is an effective approach for a group of players converging to a stable equilibrium after a period of strategic interactions, and such a stable equilibrium is called as evolutionarily stable strategy (ESS). According to the evolutionary game theory [22], for a game with $M$ players, a strategy profile $\mathbf{a}^{*}=\left(a_{1}^{*}, \ldots, a_{M}^{*}\right)$ is an ESS if and only if, $\forall \mathbf{a} \neq \mathbf{a}^{*}, \mathbf{a}^{*}$ satisfies follows:

$$
\begin{aligned}
& \text { 1) } U_{i}\left(a_{i}, \mathbf{a}_{-i}^{*}\right) \leq U_{i}\left(a_{i}^{*}, \mathbf{a}_{-i}^{*}\right), \\
& \text { 2) if } U_{i}\left(a_{i}, \mathbf{a}_{-i}^{*}\right)=U_{i}\left(a_{i}^{*}, \mathbf{a}_{-i}^{*}\right), \\
& U_{i}\left(a_{i}, \mathbf{a}_{-i}\right)<U_{i}\left(a_{i}^{*}, \mathbf{a}_{-i}\right),
\end{aligned}
$$

where $U_{i}$ stands for the utility of player $i$ and $\mathbf{a}_{-i}$ denotes the strategies of all players other than player $i$. We can see that the first condition is the Nash equilibrium (NE) condition, and the second condition guarantees the stability of the strategy. Moreover, we can also see that a strict NE is always an ESS.

2) Replicator Dynamics: In a distributed scheme, all players are uncertain about other players' actions and utilities. To improve his/her own utility, each player will try different strategies in different rounds of play and learn from the interactions using the methodology of understanding-by-building. During this process, the portion of players using a certain pure strategy may vary with time. In the evolutionary game, replicator dynamics are used to model such a population evolution. In our system, there are two strategy sets for the SUs: one is spectrum sensing strategy set $\Lambda_{1}=(s, \bar{s})$ where strategy $s$ denotes sensing and strategy $\bar{s}$ denotes not sensing, the other is spectrum access strategy set $\Lambda_{2}=(a, \bar{a})$ where strategy $a$ denotes access and strategy $\bar{a}$ denotes not access. Let $p_{s}$ denote the portion of SUs who sense the primary channel, and $p_{a}$ denote the portion of SUs who access the channel if they observe that the PU is absent after cooperative 
spectrum sensing, i.e., $\left.p(a)\right|_{\mathcal{D}_{0}}=p_{a}$ and $\left.p(a)\right|_{\mathcal{D}_{1}}=0$. Then, the evolution dynamics of $p_{s}$ and $p_{a}$ are given by

$$
\begin{aligned}
& \dot{p_{s}}=\eta p_{s}\left(\mathbb{U}_{s}-\mathbb{U}\right), \\
& \dot{p_{a}}=\eta p_{a}\left(\left.\mathbb{U}_{a}\right|_{\mathcal{D}_{0}}-\left.\mathbb{U}\right|_{\mathcal{D}_{0}}\right),
\end{aligned}
$$

where $\mathbb{U}_{s}$ is the average utility of the SUs who participate in the cooperative spectrum sensing, $\mathbb{U}$ is the average utility of all SUs, $\left.\mathbb{U}_{a}\right|_{\mathcal{D}_{0}}$ is the average utility of the SUs who access the primary channel given the condition of $\mathcal{D}_{0},\left.\mathbb{U}\right|_{\mathcal{D}_{0}}$ is the average utility of all SUs given $\mathcal{D}_{0}$, and $\eta$ is a positive scale factor. From (7), we can see that if spectrum sensing can lead to a higher utility than the average level, the portion $p_{s}$ will increase and the increasing rate $\dot{p}_{s} / p_{s}$ is proportional to the difference between $\mathbb{U}_{s}$ and $\mathbb{U}$. Similar phenomenon can be found for the evolution of $p_{a}$.

3) Utility Functions: Since we are considering the joint spectrum sensing and access game, the utility functions are determined by both $p_{s}$ and $p_{a}$. When the PU is absent, i.e., given $\mathcal{H}_{0}$, the utility functions of the SUs with four difference actions $\{s a, \bar{s} a, s \bar{a}, \overline{s a}\}$, can be written as follows:

$$
\begin{aligned}
\left.U_{s a}\right|_{\mathcal{H}_{0}} & =\mathbb{F}\left(M p_{a}\right)-\Theta_{a}-\Theta_{s}, \\
\left.U_{\bar{s} a}\right|_{\mathcal{H}_{0}} & =\mathbb{F}\left(M p_{a}\right)-\Theta_{a}, \\
\left.U_{s \bar{a}}\right|_{\mathcal{H}_{0}} & =-\Theta_{s}+R, \\
\left.U_{\overline{s a}}\right|_{\mathcal{H}_{0}} & =0,
\end{aligned}
$$

where $\mathbb{F}(\cdot)$ is the reward of a SU obtained from channel access, $\Theta_{a}=T_{a} E_{2}$ is the energy consumed by data transmission, $\Theta_{s}=T_{s} E_{3}$ is the energy consumed by spectrum sensing, and the constant $R$ is the energy reward to the $\mathrm{SU}$ who contributes to channel sensing but do not access the channel and $R>\Theta_{s}$. In the practical cognitive radio network, the energy reward $R$ can be the credit of the SUs, or a period of network access time free of charge. Here $\mathbb{F}\left(M p_{a}\right)$ represents the throughput of a SU given by

$$
\mathbb{F}\left(M p_{a}\right)=B \log \left(1+\frac{\mathrm{SNR}}{\left(M p_{a}-1\right) \cdot \mathrm{INR}+1}\right) \cdot T_{a} E_{1},
$$

where $M p_{a}$ denotes the number of SUs that choose to access the channel given $\mathcal{D}_{0}, B$ is the bandwidth of the primary channel, INR is the interference-noise ratio from each of other SUs where we assume that the SUs cause same amount of interference to each other, and $E_{1}$ is the parameter that translates one SU's throughput reward into its energy reward.

Similar to the case $\mathcal{H}_{0}$, we can summarize the SUs' utility functions under $\mathcal{H}_{1}$ as follows:

$$
\begin{aligned}
& \left.U_{s a}\right|_{\mathcal{H}_{1}}=-\Theta_{a}-\Theta_{s}, \\
& \left.U_{\bar{s} a}\right|_{\mathcal{H}_{1}}=-\Theta_{a}, \\
& \left.U_{s \bar{a}}\right|_{\mathcal{H}_{1}}=-\Theta_{s}+R, \\
& \left.U_{\overline{s a}}\right|_{\mathcal{H}_{1}}=0,
\end{aligned}
$$

where we assume that the SUs cannot obtain reward by access under $\mathcal{H}_{1}$ due to the presence of the PU.

\section{B. Replicator Dynamics of Spectrum Sensing}

The replicator dynamics of spectrum sensing are given in (7), where we need to derive the average utility of the SUs who perform channel sensing $\mathbb{U}_{s}$ and the average utility of all SUs $\mathbb{U}$. According to utility functions (9)-(17), we can calculate the average utility of performing sensing and not performing sensing given $\mathcal{H}_{0}$ or $\mathcal{H}_{1}$ as follows:

$$
\begin{aligned}
& \left.\mathbb{U}_{s}\right|_{\mathcal{H}_{0}}=\left.\left.p(a)\right|_{\mathcal{H}_{0}} \cdot U_{s a}\right|_{\mathcal{H}_{0}}+\left.\left.p(\bar{a})\right|_{\mathcal{H}_{0}} \cdot U_{s \bar{a}}\right|_{\mathcal{H}_{0}}, \\
& \left.\mathbb{U}_{s}\right|_{\mathcal{H}_{1}}=\left.\left.p(a)\right|_{\mathcal{H}_{1}} \cdot U_{s a}\right|_{\mathcal{H}_{1}}+\left.\left.p(\bar{a})\right|_{\mathcal{H}_{1}} \cdot U_{s \bar{a}}\right|_{\mathcal{H}_{1}}, \\
& \left.\mathbb{U}_{\bar{s}}\right|_{\mathcal{H}_{0}}=\left.\left.p(a)\right|_{\mathcal{H}_{0}} \cdot U_{\bar{s} a}\right|_{\mathcal{H}_{0}}+\left.\left.p(\bar{a})\right|_{\mathcal{H}_{0}} \cdot U_{\overline{s a}}\right|_{\mathcal{H}_{0}}, \\
& \left.\mathbb{U}_{\bar{s}}\right|_{\mathcal{H}_{1}}=\left.\left.p(a)\right|_{\mathcal{H}_{1}} \cdot U_{\bar{s} a}\right|_{\mathcal{H}_{1}}+\left.\left.p(\bar{a})\right|_{\mathcal{H}_{1}} \cdot U_{\overline{s a}}\right|_{\mathcal{H}_{1}},
\end{aligned}
$$

where $\left.p(a)\right|_{\mathcal{H}_{0}}$ and $\left.p(a)\right|_{\mathcal{H}_{1}}$ denote the portion of SUs who access the primary channel given channel condition $\mathcal{H}_{0}$ and $\mathcal{H}_{1}$, respectively, which can be calculated by

$$
\begin{aligned}
\left.p(a)\right|_{\mathcal{H}_{0}} & =\left.p\left(a, \mathcal{D}_{0}\right)\right|_{\mathcal{H}_{0}}=\left.\left.p(a)\right|_{\left(\mathcal{D}_{0}, \mathcal{H}_{0}\right)} \cdot p\left(\mathcal{D}_{0}\right)\right|_{\mathcal{H}_{0}}, \\
& =p_{a}\left(1-P_{f}\left(M p_{s}\right)\right), \\
\left.p(a)\right|_{\mathcal{H}_{1}} & =\left.p\left(a, \mathcal{D}_{0}\right)\right|_{\mathcal{H}_{1}}=\left.\left.p(a)\right|_{\left(\mathcal{D}_{0}, \mathcal{H}_{1}\right)} \cdot p\left(\mathcal{D}_{0}\right)\right|_{\mathcal{H}_{1}}, \\
& =p_{a}\left(1-P_{d}\right),
\end{aligned}
$$

where $P_{f}\left(M p_{s}\right)$ is the false-alarm probability when $M p_{s}$ SUs cooperatively sense the primary channel.

Thus, we can derive the average utility of SUs who sense the primary channel, $\mathbb{U}_{s}$, the average utility of SUs who do not sense, $\mathbb{U}_{\bar{s}}$, and the average utility of all SUs, $\mathbb{U}$, as follows:

$$
\begin{aligned}
\mathbb{U}_{s} & =\left.p_{0} \cdot \mathbb{U}_{s}\right|_{\mathcal{H}_{0}}+\left.p_{1} \cdot \mathbb{U}_{s}\right|_{\mathcal{H}_{1}}, \\
\mathbb{U}_{\bar{s}} & =\left.p_{0} \cdot \mathbb{U}_{\bar{s}}\right|_{\mathcal{H}_{0}}+\left.p_{1} \cdot \mathbb{U}_{\bar{s}}\right|_{\mathcal{H}_{1}}, \\
\mathbb{U} & =p_{s} \cdot \mathbb{U}_{s}+\left(1-p_{s}\right) \cdot \mathbb{U}_{\bar{s}},
\end{aligned}
$$

where $p_{0}$ is the probability that the $\mathrm{PU}$ is absent, i.e., the probability of $\mathcal{H}_{0}$, and $p_{1}=1-p_{0}$ is the probability that the PU is present, i.e., the probability of $\mathcal{H}_{1}$. Combining (7) and (9)-(26), we can re-write the replicator dynamics of spectrum sensing by (27) below.

\section{Replicator Dynamics of Spectrum Access}

Similar to the analysis of replicator dynamics of spectrum sensing, we should first calculate the average utility of SUs accessing the primary channel $\left.\mathbb{U}_{a}\right|_{\mathcal{D}_{0}}$ and the average utility of all SUs given $\mathcal{D}_{0},\left.\mathbb{U}\right|_{\mathcal{D}_{0}}$. The SUs' utilities with four pure strategies $\{s a, \bar{s} a, s \bar{a}, \overline{s a}\}$ given $\mathcal{D}_{0}$ can be written as (28)(31) below.

where $\left.p\left(\mathcal{H}_{0}\right)\right|_{\left(a, \mathcal{D}_{0}\right)}$ and $\left.p\left(\mathcal{H}_{0}\right)\right|_{\left(\bar{a}, \mathcal{D}_{0}\right)}$ are the probabilities that the PU is absent when the SUs decide to access and not access, respectively, $U . .\left.\right|_{\left(\mathcal{D}_{0}, \mathcal{H}_{0}\right)}$ and $U . .\left.\right|_{\left(\mathcal{D}_{0}, \mathcal{H}_{1}\right)}$ are the SUs' utility functions when given $\left\{\mathcal{D}_{0}, \mathcal{H}_{0}\right\}$ and $\left\{\mathcal{D}_{0}, \mathcal{H}_{1}\right\}$, respectively. Since given $\mathcal{D}_{0}$, the accessing behavior is independent with the status of the PU, we have $\left.p\left(\mathcal{H}_{0}\right)\right|_{\left(a, \mathcal{D}_{0}\right)}=$

$$
\dot{p}_{s}=\eta p_{s}\left(1-p_{s}\right)\left(\mathbb{U}_{s}-\mathbb{U}_{\bar{s}}\right)=\eta p_{s}\left(1-p_{s}\right)\left(-\Theta_{s}+\left(1-p_{a}+p_{a}\left(p_{0} P_{f}\left(M p_{s}\right)+p_{1} P_{d}\right)\right) R\right) .
$$




$$
\begin{aligned}
& \left.U_{s a}\right|_{\mathcal{D}_{0}}=\left.\left.p\left(\mathcal{H}_{0}\right)\right|_{\left(a, \mathcal{D}_{0}\right)} \cdot U_{s a}\right|_{\left(\mathcal{D}_{0}, \mathcal{H}_{0}\right)}+\left.\left(1-\left.p\left(\mathcal{H}_{0}\right)\right|_{\left(a, \mathcal{D}_{0}\right)}\right) \cdot U_{s a}\right|_{\left(\mathcal{D}_{0}, \mathcal{H}_{1}\right)}, \\
& \left.U_{\bar{s} a}\right|_{\mathcal{D}_{0}}=\left.\left.p\left(\mathcal{H}_{0}\right)\right|_{\left(a, \mathcal{D}_{0}\right)} \cdot U_{\bar{s} a}\right|_{\left(\mathcal{D}_{0}, \mathcal{H}_{0}\right)}+\left.\left(1-\left.p\left(\mathcal{H}_{0}\right)\right|_{\left(a, \mathcal{D}_{0}\right)}\right) \cdot U_{\bar{s} a}\right|_{\left(\mathcal{D}_{0}, \mathcal{H}_{1}\right)}, \\
& \left.U_{s \bar{a}}\right|_{\mathcal{D}_{0}}=\left.\left.p\left(\mathcal{H}_{0}\right)\right|_{\left(\bar{a}, \mathcal{D}_{0}\right)} \cdot U_{s \bar{a}}\right|_{\left(\mathcal{D}_{0}, \mathcal{H}_{0}\right)}+\left.\left(1-\left.p\left(\mathcal{H}_{0}\right)\right|_{\left(\bar{a}, \mathcal{D}_{0}\right)}\right) \cdot U_{s \bar{a}}\right|_{\left(\mathcal{D}_{0}, \mathcal{H}_{1}\right)}, \\
& \left.U_{\overline{s a}}\right|_{\mathcal{D}_{0}}=\left.\left.p\left(\mathcal{H}_{0}\right)\right|_{\left(\bar{a}, \mathcal{D}_{0}\right)} \cdot U_{\overline{s a}}\right|_{\left(\mathcal{D}_{0}, \mathcal{H}_{0}\right)}+\left.\left(1-\left.p\left(\mathcal{H}_{0}\right)\right|_{\left(\bar{a}, \mathcal{D}_{0}\right)}\right) \cdot U_{\overline{s a}}\right|_{\left(\mathcal{D}_{0}, \mathcal{H}_{1}\right)} .
\end{aligned}
$$

$\left.p\left(\mathcal{H}_{0}\right)\right|_{\left(\bar{a}, \mathcal{D}_{0}\right)}=\left.p\left(\mathcal{H}_{0}\right)\right|_{\mathcal{D}_{0}}$, where $\left.p\left(\mathcal{H}_{0}\right)\right|_{\mathcal{D}_{0}}$ can be calculated by the Bayes' rule given as

$$
\begin{aligned}
\left.p\left(\mathcal{H}_{0}\right)\right|_{\mathcal{D}_{0}} & =\frac{\left.p_{0} \cdot p\left(\mathcal{D}_{0}\right)\right|_{\mathcal{H}_{0}}}{\left.p_{0} \cdot p\left(\mathcal{D}_{0}\right)\right|_{\mathcal{H}_{0}}+\left.p_{1} \cdot p\left(\mathcal{D}_{0}\right)\right|_{\mathcal{H}_{1}}}, \\
& =\frac{p_{0}\left(1-P_{f}\left(M p_{s}\right)\right)}{1-p_{0} P_{f}\left(M p_{s}\right)-p_{1} P_{d}}
\end{aligned}
$$

Given the actions, the SUs' utilities are independent with $\mathcal{D}_{0}$, i.e., $\left.U_{. .}\right|_{\left(\mathcal{D}_{0}, \mathcal{H}_{0}\right)}=U . .\left.\right|_{\mathcal{H}_{0}}$ and $U . .\left.\right|_{\left(\mathcal{D}_{0}, \mathcal{H}_{1}\right)}=U . .\left.\right|_{\mathcal{H}_{1}}$. In such a case, we can derive the average utilities of SUs who access and who do not access the primary channel $\left.\mathbb{U}_{a}\right|_{\mathcal{D}_{0}}$ and $\left.\mathbb{U}_{\bar{a}}\right|_{\mathcal{D}_{0}}$, and the average utility of all SUs $\left.\mathbb{U}\right|_{\mathcal{D}_{0}}$ as follows:

$$
\begin{aligned}
\left.\mathbb{U}_{a}\right|_{\mathcal{D}_{0}} & =\left.p_{s} \cdot U_{s a}\right|_{\mathcal{D}_{0}}+\left.\left(1-p_{s}\right) \cdot U_{\bar{s} a}\right|_{\mathcal{D}_{0}}, \\
\left.\mathbb{U}_{\bar{a}}\right|_{\mathcal{D}_{0}} & =\left.p_{s} \cdot U_{s \bar{a}}\right|_{\mathcal{D}_{0}}+\left.\left(1-p_{s}\right) \cdot U_{\overline{s a}}\right|_{\mathcal{D}_{0}}, \\
\left.\mathbb{U}\right|_{\mathcal{D}_{0}} & =\left.p_{a} \cdot \mathbb{U}_{a}\right|_{\mathcal{D}_{0}}+\left.\left(1-p_{a}\right) \cdot \mathbb{U}_{\bar{a}}\right|_{\mathcal{D}_{0}} .
\end{aligned}
$$

Combining (8) and (28)-(35), we can re-write the replicator dynamics of spectrum access as (36) below.

\section{Analysis of Evolutionarily Stable Strategy}

At equilibrium, we have $\dot{p}_{s}=0$ and $\dot{p_{a}}=0$. According to (27) and (36), we can get seven possible equilibria: $(0,0),(0,1),(1,0),(1,1),\left(p_{s_{1}}, 1\right),\left(1, p_{a_{1}}\right),\left(p_{s_{2}}, p_{a_{2}}\right)$, where $p_{s_{1}}$ satisfies $P_{f}\left(M p_{s_{1}}\right)=\left(\frac{\Theta_{s}}{R}-p_{1} P_{d}\right) / p_{0}, p_{a_{1}}$ satisfies $\mathbb{F}\left(M p_{a_{1}}\right)=\frac{\left(\Theta_{a}+R\right)\left(1-p_{0} P_{f}(M)-p_{1} P_{d}\right)}{p_{0}\left(1-P_{f}(M)\right)}$ and $\left(p_{s_{2}}, p_{a_{2}}\right)$ is the solution to the following equations

$$
\left\{\begin{array}{l}
-\Theta_{s}+\left(1-p_{a}+p_{a}\left(p_{0} P_{f}\left(M p_{s}\right)+p_{1} P_{d}\right)\right) R=0 \\
\frac{p_{0}\left(1-P_{f}\left(M p_{s}\right)\right)}{1-p_{0} P_{f}\left(M p_{s}\right)-p_{1} P_{d}} \mathbb{F}\left(M p_{a}\right)-\Theta_{a}-p_{s} R=0 .
\end{array}\right.
$$

According to the evolutionary game theory [22], an equilibrium of the replicator dynamics equation is an ESS if and if only it is a locally asymptotically stable point in a dynamic system. In the following Lemma 1 and Theorem 1, we will check which equilibria are the ESSs.

Lemma 1: The false-alarm probability $P_{f}$ is a decreasing function in terms of $p_{s}$, and the reward from channel access $\mathbb{F}$ is a decreasing function in terms of $p_{a}$, i.e., $\frac{d P_{f}\left(M p_{s}\right)}{d p_{s}}<0$ and $\frac{d \mathbb{F}\left(M p_{a}\right)}{d p_{a}}<0$.

Proof: According to (4), we have

$$
\frac{d P_{f}\left(M p_{s}\right)}{d p_{s}}=-\frac{\gamma}{2 \sqrt{\pi}} \sqrt{\frac{\lambda T_{s} M}{2 p_{s}}} e^{-\left(\Omega+\sqrt{\frac{\lambda T_{s} M p_{s}}{2}} \gamma\right)^{2}}<0 .
$$

According to (13) and (38), we have

$$
\begin{aligned}
\frac{d \mathbb{F}\left(M p_{a}\right)}{d p_{a}}= & -\frac{B \cdot T_{a} \cdot E_{1}}{\left(M p_{a}-1\right) \mathrm{INR}+1+\mathrm{SNR}} . \\
& \frac{M \cdot \mathrm{SNR} \cdot \mathrm{INR}}{\left(M p_{a}-1\right) \mathrm{INR}+1}<0 .
\end{aligned}
$$

Eq. (38) means that the larger $p_{s}$ is, the more SUs contribute the cooperative spectrum sensing and the lower $P_{f}$ can be achieved, while the physical meaning of (39) is that the more SUs access the primary channel, the less reward can be obtained from channel access by each SU.

Theorem 1: For the joint spectrum sensing and access evolutionary game under the synchronous scenario, there are three ESSs: $\left(p_{s}^{*}, p_{a}^{*}\right)=(1,0),\left(1, p_{a_{1}}\right)$ and $\left(p_{s_{2}}, p_{a_{2}}\right)$, under different conditions of the rewards $R$ as (40) below.

Proof: Considering (27) and (36) as a nonlinear dynamic system, we can examine whether the seven equilibria are ESSs through analyzing the Jacobian matrix of the replicator dynamics equations as follows:

$$
J=\left(\begin{array}{ll}
\frac{\partial \dot{p}_{s}}{\partial p_{s}} & \frac{\partial \dot{p}_{s}}{\partial p_{a}} \\
\frac{\partial \dot{p}_{a}}{\partial p_{s}} & \frac{\partial \dot{p}_{a}}{\partial p_{a}}
\end{array}\right)=\eta\left(\begin{array}{cc}
J_{11} & J_{12} \\
J_{21} & J_{22}
\end{array}\right)
$$

where $J_{11}, J_{12}, J_{21}$ and $J_{22}$ are listed in (42)-(45) below.

$$
\begin{gathered}
\dot{p}_{a}=\eta p_{a}\left(1-p_{a}\right)\left(\left.\mathbb{U}_{a}\right|_{\mathcal{D}_{0}}-\left.\mathbb{U}_{\bar{a}}\right|_{\mathcal{D}_{0}}\right)=\eta p_{a}\left(1-p_{a}\right)\left(\frac{p_{0}\left(1-P_{f}\left(M p_{s}\right)\right)}{1-p_{0} P_{f}\left(M p_{s}\right)-p_{1} P_{d}} \mathbb{F}\left(M p_{a}\right)-\Theta_{a}-p_{s} R\right) . \\
\left(p_{s}^{*}, p_{a}^{*}\right)= \begin{cases}(1,0), & R>\frac{p_{0}\left(1-P_{f}(M)\right)}{1-p_{0} P_{f}(M)-p_{1} P_{d}} \mathbb{F}(1)-\Theta_{a} \\
\left(1, p_{a_{1}}\right), & R>\frac{\Theta_{s}}{1-p_{a_{1}}\left(1-p_{0} P_{f}(M)-p_{1} P_{d}\right)}, \\
\left(p_{s_{2}}, p_{a_{2}}\right), & R<\frac{p_{0}^{2} p_{s_{2}}\left(1-P_{f}\left(M p_{s_{2}}\right)\right) \frac{d \mathbb{F}\left(M p_{a_{2}}\right)}{d p_{a_{2}}}-\frac{p_{0} p_{1}\left(1-P_{d}\right) \mathbb{F}\left(M p_{a_{2}}\right)}{1-p_{0} P_{f}\left(M p_{s_{2}}\right)-p_{1} P_{d}}}{\left(1-p_{0} P_{f}\left(M p_{s_{2}}\right)-p_{1} P_{d}\right)^{2}} \frac{d P_{f}\left(M p_{s_{2}}\right)}{d p_{s_{2}}}\end{cases}
\end{gathered}
$$




$$
\begin{aligned}
& J_{11}=\left(1-2 p_{s}\right)\left(-\Theta_{s}+\left(1-p_{a}+p_{a}\left(p_{0} P_{f}\left(M p_{s}\right)+p_{1} P_{d}\right)\right) R\right)+p_{0} p_{s}\left(1-p_{s}\right) p_{a} \frac{d P_{f}\left(M p_{s}\right)}{d p_{s}} R, \\
& J_{12}=-p_{s}\left(1-p_{s}\right)\left(1-p_{0} P_{f}\left(M p_{s}\right)-p_{1} P_{d}\right) R, \\
& J_{21}=p_{a}\left(1-p_{a}\right)\left(-\frac{p_{0} p_{1}\left(1-P_{d}\right) \mathbb{F}\left(M p_{a}\right)}{\left(1-p_{0} P_{f}\left(M p_{s}\right)-p_{1} P_{d}\right)^{2}} \frac{d P_{f}\left(M p_{s}\right)}{d p_{s}}-R\right), \\
& J_{22}=\left(1-2 p_{a}\right)\left(\frac{p_{0}\left(1-P_{f}\left(M p_{s}\right)\right) \mathbb{F}\left(M p_{a}\right)}{1-p_{0} P_{f}\left(M p_{s}\right)-p_{1} P_{d}}-\Theta_{a}-p_{s} R\right)+p_{0} \frac{p_{a}\left(1-p_{a}\right)\left(1-P_{f}\left(M p_{s}\right)\right)}{1-p_{0} P_{f}\left(M p_{s}\right)-p_{1} P_{d}} \frac{d \mathbb{F}\left(M p_{a}\right)}{d p_{a}} . \\
& \quad \begin{array}{c}
\operatorname{det}(J)= \\
\quad \frac{p_{s_{1}}\left(1-p_{s_{1}}\right) p_{a_{1}}\left(1-p_{a_{1}}\right) R}{1-p_{0} P_{f}\left(M p_{s_{2}}\right)-p_{1} P_{d}}\left(p_{0}^{2} p_{a_{2}}\left(1-P_{f}\left(M p_{s_{2}}\right)\right) \frac{d \mathbb{F}\left(M p_{a_{2}}\right)}{d p_{a_{2}}} \frac{d P_{f}\left(M p_{s_{2}}\right)}{d p_{s_{2}}}\right. \\
\left.\quad-\frac{p_{0} p_{1}\left(1-P_{d}\right) \mathbb{F}\left(M p_{s_{2}}\right)}{1-p_{0} P_{f}\left(M p_{s_{2}}\right)-p_{1} P_{d}} \frac{d P_{f}\left(M p_{s_{2}}\right)}{d p_{s_{2}}}-\left(1-p_{0} P_{f}\left(M p_{s_{2}}\right)-p_{1} P_{d}\right)^{2} R\right) .
\end{array}
\end{aligned}
$$

The asymptotical stability requires that $\operatorname{det}(J)>0$ and $\operatorname{tr}(J)<0$ [22]. Substituting the seven equilibria into (41) separately, we can examine whether they are ESSs. For the equilibrium $(0,0)$, i.e., no $\mathrm{SU}$ senses and accesses the primary channel, we have $\lim _{p_{s} \rightarrow 0} J_{12} \cdot J_{21}=0$ since

$$
\begin{aligned}
& \lim _{p_{s} \rightarrow 0} p_{s} \frac{d P_{f}\left(M p_{s}\right)}{d p_{s}} \\
= & \lim _{p_{s} \rightarrow 0}-\frac{\gamma}{2 \sqrt{\pi}} \sqrt{\frac{\lambda T_{s} M p_{s}}{2}} e^{-\left(\Omega+\sqrt{\frac{\lambda T_{s} M p_{s}}{2}} \gamma\right)^{2}}=0 .
\end{aligned}
$$

In such a case, $(0,0)$ is an ESS if and only if $\lim _{p_{s} \rightarrow 0} J_{11}<0$ and $\lim _{p_{s} \rightarrow 0} J_{22}<0$. However, $\lim _{p_{s} \rightarrow 0} J_{11}=-\Theta_{s}+\stackrel{p_{s} \rightarrow 0}{>}>0$. Therefore, $(0,0)$ is not an ESS. Similarly, the equilibrium $(0,1)$, i.e., no SU senses but all SUs access the primary channel, is not an ESS because when $p_{a}=1$, the channel will be extremely crowed and $\mathbb{F}(M) \rightarrow 0$, resulting in $J_{22} \rightarrow \Theta_{a}>0$. Moreover, the equilibrium $(1,1)$, i.e., all SUs sense and access the channel, and the equilibrium $\left(p_{s_{1}}, 1\right)$, i.e., part of SUs sense but all SUs access the channel, are neither ESSs because $J_{22} \rightarrow \Theta_{a}+R>0$ and $J_{12} \cdot J_{21}=0$.

For the equilibrium $(1,0)$ which means all SUs sense but no SU access the channel, we have

$$
\begin{aligned}
& \lim _{p_{a} \rightarrow 0} J_{11}=\Theta_{s}-R<0, \\
& \lim _{p_{a} \rightarrow 0} J_{22}=\frac{p_{0}\left(1-P_{f}(M)\right)}{1-p_{0} P_{f}(M)-p_{1} P_{d}} \mathbb{F}(1)-\Theta_{a}-R, \\
& \lim _{p_{a} \rightarrow 0} J_{12} \cdot J_{21}=0 .
\end{aligned}
$$

Therefore, $(1,0)$ is an ESS when the reward $R$ satisfies

$$
R>\frac{p_{0}\left(1-P_{f}(M)\right)}{1-p_{0} P_{f}(M)-p_{1} P_{d}} \mathbb{F}(1)-\Theta_{a} .
$$

From (51), we can see that if the reward for an SU who senses but does not access, $R$, is larger than the reward the SU can obtain by solely occupying the channel, $\left.p\left(\mathcal{H}_{0}\right)\right|_{\mathcal{D}_{0}} \cdot \mathbb{F}(1)-$ $\Theta_{a}$ according to (32), then all SUs would choose to perform sensing but not to access the channel. Such an ESS would lead to a low utilization of the spectrum. Therefore, $R$ should be appropriately set to avoid the system state converging into such an undesired ESS.

For the equilibrium $\left(1, p_{a_{1}}\right)$, i.e., all SUs sense but only part of SUs choose to access the primary channel, we have $J_{12} \cdot J_{21}=0$ and according to Lemma 1

$$
\begin{aligned}
& J_{11}=\Theta_{s}-\left(1-p_{a_{1}}\left(1-p_{0} P_{f}(M)-p_{1} P_{d}\right)\right) R, \\
& J_{22}=\frac{p_{0} p_{a_{1}}\left(1-p_{a_{1}}\right)\left(1-P_{f}(M)\right)}{1-p_{0} P_{f}(M)-p_{1} P_{d}} \frac{d \mathbb{F}\left(M p_{a_{2}}\right)}{d p_{a_{2}}}<0 .
\end{aligned}
$$

In such a case, $\left(1, p_{a_{1}}\right)$ is an ESS if the reward $R$ satisfies

$$
R>\frac{\Theta_{s}}{1-p_{a_{1}}\left(1-p_{0} P_{f}(M)-p_{1} P_{d}\right)} .
$$

For the solution $\left(p_{s_{2}}, p_{a_{2}}\right)$, i.e., part of SUs sense and access the primary channel, we have

$$
\begin{aligned}
J_{11}= & p_{0} p_{s_{2}}\left(1-p_{s_{2}}\right) p_{a_{2}} \frac{d P_{f}\left(M p_{s_{2}}\right)}{d p_{s_{2}}} R<0, \\
J_{12}= & -p_{s_{2}}\left(1-p_{s_{2}}\right)\left(1-p_{0} P_{f}\left(M p_{s_{2}}\right)-p_{1} P_{d}\right) R, \\
J_{21}= & p_{a_{2}}\left(1-p_{a_{2}}\right)\left(-\frac{p_{0} p_{1}\left(1-P_{d}\right) \mathbb{F}\left(M p_{a_{2}}\right)}{\left(1-p_{0} P_{f}\left(M p_{a_{2}}\right)-p_{1} P_{d}\right)^{2}}\right. \\
& \left.\frac{d P_{f}\left(M p_{s_{2}}\right)}{d p_{s_{2}}}-R\right), \\
J_{22}= & p_{0} \frac{p_{a_{2}}\left(1-p_{a_{2}}\right)\left(1-P_{f}\left(M p_{s_{2}}\right)\right)}{1-p_{0} P_{f}\left(M p_{a_{2}}\right)-p_{1} P_{d}} \frac{d \mathbb{F}\left(M p_{a_{2}}\right)}{d p_{a_{2}}}<0
\end{aligned}
$$

With (55)-(58), we can calculate $\operatorname{det}(J)$ in (46) above. Thus, $\left(p_{s_{2}}, p_{a_{2}}\right)$ is an ESS when the reward $R$ satisfies

$$
\begin{aligned}
R< & \frac{p_{0}^{2} p_{s_{2}}\left(1-P_{f}\left(M p_{s_{2}}\right)\right) \frac{d \mathbb{F}\left(M p_{a_{2}}\right)}{d p_{a_{2}}}-\frac{p_{0} p_{1}\left(1-P_{d}\right) \mathbb{F}\left(M p_{a_{2}}\right)}{1-p_{0} P_{f}\left(M p_{s_{2}}\right)-p_{1} P_{d}}}{\left(1-p_{0} P_{f}\left(M p_{s_{2}}\right)-p_{1} P_{d}\right)^{2}} \\
& \frac{d P_{f}\left(M p_{s_{2}}\right)}{d p_{s_{2}}} .
\end{aligned}
$$

To summarize, (51), (54) and (59) are the conditions for the three $\operatorname{ESSs}\left(p_{s}^{*}, p_{a}^{*}\right)=(1,0),\left(1, p_{a_{1}}\right)$ and $\left(p_{s_{2}}, p_{a_{2}}\right)$, respectively. This completes the proof of the theorem. 


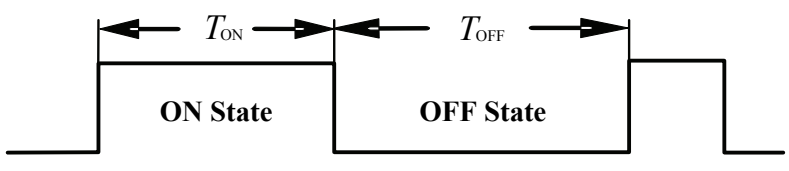

Fig. 3. Illustration of the ON-OFF primary channel state.

\section{EVOLUTIONARY GAME FORMULATION FOR ASYNCHRONOUS SCENARIO}

In this section, we will discuss the scenario when the SUs are asynchronous with the PU. The SUs' behavior is similar to that in the synchronous scenario: if they observe that the PU is absent, they will access the primary channel with time $T_{a}$; otherwise, they will wait for the next slot and re-sense the channel. The difference between the synchronous and asynchronous scenarios is that in the synchronous scenario, the interference to the PU only comes from the SUs' imperfect spectrum sensing; while in the asynchronous scenario, additional interference may appear since the SUs may fail to discover the PU's recurrence when it is transmitting a packet in the primary channel, as shown by the black regions in Fig. 2(b). The essential reason is that the half-duplex SUs cannot perform sensing when transmitting data [31]. Therefore, in the asynchronous scenario, the SUs' access time $T_{a}$ should be appropriately chosen to control the interference level and guarantee the PU's QoS. In the following, we model the primary channel as an ON-OFF process and derive the proper $T_{a}$. Then, we define the utility functions of the SUs under the asynchronous scenario. Finally, we find the ESS of spectrum sensing and access.

\section{A. ON-OFF Primary Channel Model}

Since the SUs have no idea about the exact communication mechanism of the primary network and hence cannot be synchronous with the PU, there is no concept of "time slot" in the primary channel from the SUs' points of view [32]. Instead, the primary channel just alternatively switches between ON state and OFF state, as shown in Fig. 3. The ON state means the channel is occupied by the PU, while the OFF state is the "spectrum hole" which can be freely accessed by the SUs. We model the length of the ON state and OFF state by two random variables $T_{\mathrm{ON}}$ and $T_{\mathrm{OFF}}$, respectively. According to different types of the primary services (e.g., digital TV broadcasting or cellular communication), $T_{\mathrm{ON}}$ and $T_{\text {OFF }}$ statistically satisfy different distributions. Here we assume that $T_{\mathrm{ON}}$ and $T_{\mathrm{OFF}}$ are independent and satisfy exponential distributions with parameter $\lambda_{1}$ and $\lambda_{0}$, respectively, denoted by $f_{\mathrm{ON}}(t)$ and $f_{\mathrm{OFF}}(t)$ as follows:

$$
\left\{\begin{array}{l}
T_{\mathrm{ON}} \sim f_{\mathrm{ON}}(t)=\frac{1}{\lambda_{1}} e^{-t / \lambda_{1}}, \\
T_{\mathrm{OFF}} \sim f_{\mathrm{OFF}}(t)=\frac{1}{\lambda_{0}} e^{-t / \lambda_{0}} .
\end{array}\right.
$$

In such a case, the expected lengths of the ON state and OFF state are $\lambda_{1}$ and $\lambda_{0}$ accordingly. These two parameters $\lambda_{1}$ and $\lambda_{0}$ can be effectively estimated by a maximum likelihood estimator [33]. Such an ON-OFF behavior of the PU is a combination of two Poisson process, which is a renewal process [34]. The renewal interval is $T_{p}=T_{\mathrm{ON}}+T_{\mathrm{OFF}}$ and the distribution of $T_{p}$, denoted by $f_{p}(t)$, is

$$
f_{p}(t)=f_{\mathrm{ON}}(t) * f_{\mathrm{OFF}}(t),
$$

where the symbol " $*$ " represents the convolution operation. Thus, we have $\mu_{1}=\lambda_{1} /\left(\lambda_{0}+\lambda_{1}\right)$ is the occurrence probability of the ON state, also called as channel utilization ratio. Similarly, $\mu_{0}=\lambda_{0} /\left(\lambda_{0}+\lambda_{1}\right)$ is the occurrence probability of the OFF state.

\section{B. Analysis of SUs' Access Time $T_{a}$}

For the SUs, they always want to access the primary channel more time to obtain more throughput. However, the larger $T_{a}$ is, the more interference will be caused to the PU. To find a proper $T_{a}$ for both SUs and the PU, we first introduce a variable $Q_{I}$ to quantify the interference from the SUs. The $Q_{I}$ is defined as the portion of the periods when the PU is interfered to their overall communication time, i.e., the length of all the ON states. Since the interference only appears during the SUs' access time, there are two possible cases:

1) the PU is absent, i.e., the channel is in the OFF state, the SUs successfully discover it and access the channel, but the PU is back to this channel during the SUs' access time, as shown in Fig. 2-(b),

2) the PU is present, i.e., the channel is in the ON state, the SUs failed to discover it and access.

In such a case, $Q_{I}$ can be calculated by

$$
\begin{aligned}
Q_{I} & =\lim _{N \rightarrow \infty} \frac{N \mu_{0}\left(1-P_{f}\right) I_{1}\left(T_{a}\right)+N \mu_{1}\left(1-P_{d}\right) I_{2}\left(T_{a}\right)}{N T_{a} \cdot \mu_{1}} \\
& =\frac{\mu_{0}\left(1-P_{f}\right) I_{1}\left(T_{a}\right)+\mu_{1}\left(1-P_{d}\right) I_{2}\left(T_{a}\right)}{\mu_{1} T_{a}} \\
& \leq \frac{\mu_{0} I_{1}\left(T_{a}\right)+\mu_{1}\left(1-P_{d}\right) I_{2}\left(T_{a}\right)}{\mu_{1} T_{a}}
\end{aligned}
$$

where the denominator is the expected length of all ON states during a long period of time $N T_{a}$, and the numerator is the interference caused by the SUs during $N T_{a}$. The $I_{1}\left(T_{a}\right)$ is the expected length of all ON states within time $T_{a}$, given $T_{a}$ beginning from the OFF state, while the $I_{2}\left(T_{a}\right)$ is that given $T_{a}$ beginning from the $\mathrm{ON}$ state. From the definition of $Q_{I}$, we can see that it represents the occurrence probability of interference to the PU. In such a case, the PU's average data rate $R_{a v}=\left(1-Q_{I}\right) R_{p}$, where $R_{p}$ is the PU's data rate when there is no interference from the SUs. To ensure the PU's reliable communication, a minimum average data rate $R_{a v}^{\downarrow}$ should be guaranteed, i.e., $R_{a v} \geq R_{a v}^{\downarrow}$ which means that $Q_{I} \leq 1-\frac{R_{a v}^{\downarrow}}{R_{p}}$. Combining this PU's QoS constraint with (62), we have

$$
\frac{\mu_{0} I_{1}\left(T_{a}\right)+\mu_{1}\left(1-P_{d}\right) I_{2}\left(T_{a}\right)}{\mu_{1} T_{a}}=1-\frac{R_{a v}^{\downarrow}}{R_{p}} .
$$

In our previous work [31], we have derived the closed-form expression for $I_{1}\left(T_{a}\right)$ using the renewal theory given as

$$
I_{1}\left(T_{a}\right)=\frac{\lambda_{1}}{\lambda_{0}+\lambda_{1}} T_{a}-\frac{\lambda_{0} \lambda_{1}^{2}}{\left(\lambda_{0}+\lambda_{1}\right)^{2}}\left(1-e^{-\frac{\lambda_{0}+\lambda_{1}}{\lambda_{0} \lambda_{1}} T_{a}}\right) .
$$

Similarly, the closed-form expression for $I_{2}\left(T_{a}\right)$ can also be obtained by the renewal theory. 


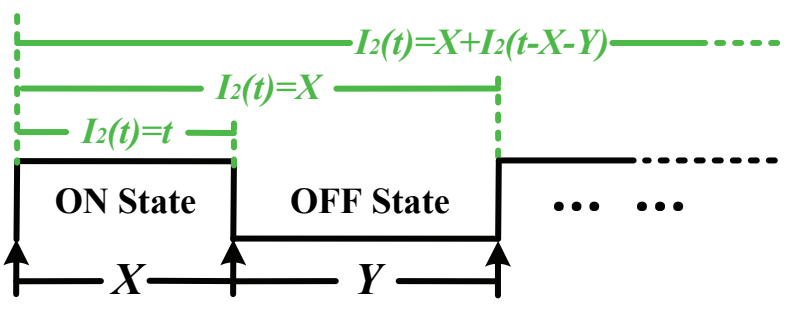

Fig. 4. Illustration of function $I_{2}(t)$.

Lemma 2: $I_{2}(t)$ satisfies the renewal equation given by

$$
I_{2}(t)=\lambda_{1} F_{\mathrm{ON}}(t)+\int_{0}^{t} I_{2}(t-w) f_{p}(w) d w,
$$

where $F_{\mathrm{ON}}(t)=\int_{0}^{t} f_{\mathrm{ON}}(t) d t=1-e^{-\frac{t}{\lambda_{1}}}$ is the cumulative distribution function (c.d.f) of the ON state and $f_{p}(t)$ is the probability density function (p.d.f) of the PU's renewal interval given in (61).

Proof: According to Fig. 4, we can first write the recursive expression of function $I_{2}(t)$ as follows:

$$
I_{2}(t)= \begin{cases}t & t \leq X, \\ X & X \leq t \leq X+Y, \\ X+I_{2}(t-X-Y) & X+Y \leq t,\end{cases}
$$

where $X$ denotes the length of the first ON state and $Y$ denotes the length of the first OFF state. Moreover, we have $X \sim$ $f_{\mathrm{ON}}(x)=\frac{1}{\lambda_{1}} e^{-x / \lambda_{1}}$ and $Y \sim f_{\mathrm{OFF}}(y)=\frac{1}{\lambda_{0}} e^{-y / \lambda_{0}}$. Since $X$ and $Y$ are independent, their joint distribution is $f_{X Y}(x, y)=$ $f_{\mathrm{ON}}(x) f_{\mathrm{OFF}}(y)$. In such a case, we can re-write $I_{2}(t)$ as follows:

$$
\begin{aligned}
I_{2}(t)= & \int_{x \geq t} t f_{\mathrm{ON}}(x) d x+\iint_{x \leq t \leq x+y} x f_{X Y}(x, y) d x d y \\
& +\iint_{x+y \leq t}\left[x+I_{2}(t-x-y)\right] f_{X Y}(x, y) d x d y, \\
= & t-\int_{0}^{t}(t-x) f_{\mathrm{ON}}(x) d x \\
& +\iint_{x+y \leq t} I_{2}(t-x-y) f_{\mathrm{ON}}(x) f_{\mathrm{OFF}}(y) d x d y \\
= & t-t * f_{\mathrm{ON}}(t)+I_{2}(t) * f_{p}(t) .
\end{aligned}
$$

By taking Laplace transforms on the both sides of (67), we have

$$
\begin{aligned}
\mathbb{I}_{2}(s) & =\frac{1}{s^{2}}-\frac{1}{s^{2}} \mathbb{F}_{\mathrm{ON}}(s)+\mathbb{I}_{2}(s) \mathbb{F}_{p}(s), \\
& =\lambda_{1} \frac{\mathbb{F}_{\mathrm{ON}}(s)}{s}+\mathbb{I}_{2}(s) \mathbb{F}_{p}(s),
\end{aligned}
$$

where $\mathbb{I}_{2}(s)$ is the Laplace transform of $I_{2}(t), \mathbb{F}_{\mathrm{ON}}(s)=\frac{1}{\lambda_{1} s+1}$ is the Laplace transform of $f_{\mathrm{oN}}(t)$, and $\mathbb{F}_{p}(s)=\frac{1}{\left(\lambda_{1} s+1\right)\left(\lambda_{0} s+1\right)}$ is the Laplace transform of $f_{p}(t)$. Then by taking the inverse Laplace transform on the both sides of (68), we have

$$
\begin{aligned}
I_{2}(t) & =\lambda_{1} \int_{0}^{t} f_{\mathrm{ON}}(w) d w+\int_{0}^{t} I_{2}(t-w) f_{p}(w) d w \\
& =\lambda_{1} F_{\mathrm{ON}}(t)+\int_{0}^{t} I_{2}(t-w) f_{p}(w) d w
\end{aligned}
$$

This completes the proof of the lemma.

Thus, by solving (68) in Lemma 2, we can obtain the closedform expression for $I_{2}\left(T_{a}\right)$ given by

$$
I_{2}\left(T_{a}\right)=\frac{\lambda_{1}}{\lambda_{0}+\lambda_{1}} T_{a}+\frac{\lambda_{0}^{2} \lambda_{1}}{\left(\lambda_{0}+\lambda_{1}\right)^{2}}\left(1-e^{-\frac{\lambda_{0}+\lambda_{1}}{\lambda_{0} \lambda_{1}} T_{a}}\right) .
$$

Substituting (64) and (70) into (63), we can obtain the proper $T_{a}$ that satisfies the PU's QoS constraint.

\section{Analysis of Evolutionarily Stable Strategy}

In the asynchronous scenario, the utility functions are different with those in the synchronous scenario. On one hand, when the PU is absent and the SUs choose to access the primary channel, they may be interfered by the PU's recurrences. On the other hand, when the PU is present but the SUs fail to detect this and access the primary channel, it may still obtain some throughput since the channel may switch into the OFF state during the SUs' access time. Taking these two cases into account, we can write the utility functions in the asynchronous scenario as follows:

$$
\begin{gathered}
\left\{\begin{array}{l}
\left.U_{s a}\right|_{\mathcal{H}_{0}}=\mathbb{W}_{1}\left(M p_{a}\right)-\Theta_{a}-\Theta_{s}, \\
\left.U_{s \bar{a}}\right|_{\mathcal{H}_{0}}=-\Theta_{s}+R, \\
\left.U_{\bar{s} a}\right|_{\mathcal{H}_{0}}=\mathbb{W}_{1}\left(M p_{a}\right)-\Theta_{a}, \\
\left.U_{\overline{s a}}\right|_{\mathcal{H}_{0}}=0,
\end{array}\right. \\
\left\{\begin{array}{l}
\left.U_{s a}\right|_{\mathcal{H}_{1}}=\mathbb{W}_{2}\left(M p_{a}\right)-\Theta_{a}-\Theta_{s}, \\
\left.U_{s \bar{a}}\right|_{\mathcal{H}_{1}}=-\Theta_{s}+R, \\
\left.U_{\bar{s} a}\right|_{\mathcal{H}_{1}}=\mathbb{W}_{2}\left(M p_{a}\right)-\Theta_{a}, \\
\left.U_{\overline{s a}}\right|_{\mathcal{H}_{1}}=0,
\end{array}\right. \\
\text { and }\left\{\begin{array}{l}
\mathbb{W}_{1}\left(M p_{a}\right)=\phi\left(M p_{a}\right)\left(T_{a}-I_{1}\left(T_{a}\right)\right) E_{1}, \\
\mathbb{W}_{2}\left(M p_{a}\right)=\phi\left(M p_{a}\right)\left(T_{a}-I_{2}\left(T_{a}\right)\right) E_{1} .
\end{array}\right.
\end{gathered}
$$

where $\phi\left(M p_{a}\right)=B \log \left(1+\frac{\mathrm{SNR}}{\left(M p_{a}-1\right) \mathrm{INR}+1}\right)$. Similar to the synchronous scenario, we can write the replicator dynamics equations of the asynchronous scenario by (74) and (75) below, where $\xi_{1}=\frac{T_{a}-I_{1}\left(T_{a}\right)}{T_{a}}$ and $\xi_{2}=\frac{T_{a}-I_{2}\left(T_{a}\right)}{T_{a}}$. When $T_{a}<<\lambda_{1}$ and $\lambda_{0}$, we can see that $I_{1}\left(T_{a}\right)$ goes to 0 and $I_{2}\left(T_{a}\right)$ goes to $T_{a}$ according to (64) and (70). In such a case, $\xi_{1} \rightarrow 1$ and $\xi_{2} \rightarrow 0$ which means that the asynchronous scenario reduces to the synchronous scenario when $T_{a}<<\lambda_{1}$ and $\lambda_{0}$. Through analyzing the replicator dynamics equations (74) and (75), which is similar to the ESS analysis in the synchronous scenario, we have the following Theorem 2.

Theorem 2: For the joint spectrum sensing and access evolutionary game under the asynchronous scenario, there are three ESSs: $\left(p_{s}^{*}, p_{a}^{*}\right)=(1,0),\left(1, p_{a_{1}}\right)$ and $\left(p_{s_{2}}, p_{a_{2}}\right)$, under different conditions of the rewards $R$ as follow (76).

\section{A Distributed Learning Algorithm for ESS}

In the above joint spectrum sensing and access evolutionary games, we have obtained the ESSs for the SUs. Thus, a group of SUs can achieve the ESS using the replicator dynamics equations (27) (36) in the synchronous scenario and (74) (75) in the asynchronous scenario. We can see that solving these equations requires the exchange of utilities among all SUs to find the average utilities such as $\mathbb{U}_{s}$ and $\left.\mathbb{U}_{a}\right|_{\mathcal{D}_{0}}$. However, in a distributed network, it is generally difficult to make each SU 


$$
\begin{aligned}
& \dot{p}_{s}=\eta p_{s}\left(1-p_{s}\right)\left(-\Theta_{s}+\left(1-p_{a}+p_{a}\left(p_{0} P_{f}+p_{1} P_{d}\right)\right) R\right), \\
& \dot{p}_{a}=\eta p_{a}\left(1-p_{a}\right)\left(\frac{p_{0}\left(1-P_{f}\left(M p_{s}\right)\right) \mathbb{W}_{1}\left(M p_{a}\right)}{1-p_{0} P_{f}\left(M p_{s}\right)-p_{1} P_{d}}+\frac{p_{1}\left(1-P_{d}\right) \mathbb{W}_{2}\left(M p_{a}\right)}{1-p_{0} P_{f}\left(M p_{s}\right)-p_{1} P_{d}}-\Theta_{a}-p_{s} R\right), \\
&=\eta p_{a}\left(1-p_{a}\right)\left(\left(\left(\xi_{1}-\xi_{2}\right) \frac{p_{0}\left(1-P_{f}\left(M p_{s}\right)\right)}{1-p_{0} P_{f}\left(M p_{s}\right)-p_{1} P_{d}}+\xi_{2}\right) \mathbb{F}\left(M p_{a}\right)-\Theta_{a}-p_{s} R\right) . \\
&\left(p_{s}^{*}, p_{a}^{*}\right)= \begin{cases}(1,0), & R>\left(\left(\xi_{1}-\xi_{2}\right) \frac{p_{0}\left(1-P_{f}(M)\right)}{1-p_{0} P_{f}(M)-p_{1} P_{d}}+\xi_{2}\right) \mathbb{F}(1)-\Theta_{a}, \\
\left(1, p_{a_{1}}\right), & R>\frac{\Theta_{s}}{1-p_{a_{1}}\left(1-p_{0} P_{f}(M)-p_{1} P_{d}\right)}, \\
\left(p_{s_{2}}, p_{a_{2}}\right), & R<\frac{p_{0}^{2} p_{s_{2}}\left(1-P_{f}\left(M p_{s_{2}}\right)\right) \frac{d \mathbb{F}\left(M p_{a_{2}}\right)}{d p_{a_{2}}}-\frac{p_{0} p_{1}\left(1-P_{d}\right) \mathbb{F}\left(M p_{a_{2}}\right)}{1-p_{0} P_{f}\left(M p_{s_{2}}\right)-p_{1} P_{d}}}{\left(1-p_{0} P_{f}\left(M p_{s_{2}}\right)-p_{1} P_{d}\right)^{2} /\left(\xi_{1}-\xi_{2}\right)} \frac{d P_{f}\left(M p_{s_{2}}\right)}{d p_{s_{2}}} .\end{cases}
\end{aligned}
$$

reveal such private information. In this section, we will present a distributed learning algorithm that can gradually converge to ESS without private utility information exchange.

In the evolutionary biology, the Wright-Fisher model has been widely used to study the population reproduction dynamics under natural selection [35]. The model is based on the assumption that the probability of an individual adopting a certain strategy is proportional to the expected utility of the population using that strategy. Let $\tilde{p}_{s}$ and $\tilde{p}_{a}$ be the probabilities of a SU sensing and accessing the primary channel, respectively. According to the Wright-Fisher model, $\tilde{p}_{s}$ is proportional to the total utility of the SUs sensing the channel. In such a case, the SUs' strategy of spectrum sensing at time slot $t+1, \tilde{p}_{s}(t+1)$, can be calculated by

$$
\begin{aligned}
\tilde{p}_{s}(t+1) & =\frac{p_{s}(t) \widetilde{\mathbb{U}}_{s}(t)}{p_{s}(t) \widetilde{\mathbb{U}}_{s}(t)+\left(1-p_{s}(t)\right) \widetilde{\mathbb{U}}_{\bar{s}}(t)}, \\
& =\frac{p_{s}(t) \widetilde{\mathbb{U}}_{s}(t)}{\widetilde{\mathbb{U}}(t)},
\end{aligned}
$$

where $\widetilde{\mathbb{U}}_{s}(t)$ and $\widetilde{\mathbb{U}}_{\bar{s}}(t)$ are the average utilities of the SUs who have sensed and not sensed the channel at the th time slot, respectively, the denominator $\widetilde{\mathbb{U}}(t)$ is the average utility of all SUs, which is the normalization term that ensures $\tilde{p}_{s}+\tilde{p}_{\bar{s}}=1$. If the SUs observe that the PU is present after cooperative spectrum sensing at the beginning of the time slot, they will always not access the primary channel within this slot. Otherwise, each SU will access the channel with probability $\tilde{p}_{a}$. With the Wright-Fisher model, $\tilde{p}_{a}$ is proportional to the total utility of the SUs who choose to access the channel. In such a case, one SU's strategy of channel access $\tilde{p}_{a}$ can be computed by

$$
\begin{aligned}
\tilde{p}_{a}\left(t^{\prime}+1\right) & =\frac{p_{a}\left(t^{\prime}\right) \widetilde{\mathbb{U}}_{a}\left(t^{\prime}\right)}{p_{a}\left(t^{\prime}\right) \widetilde{\mathbb{U}}_{a}\left(t^{\prime}\right)+\left(1-p_{a}\left(t^{\prime}\right)\right) \widetilde{\mathbb{U}}_{\bar{a}}\left(t^{\prime}\right)}, \\
& =\frac{p_{a}\left(t^{\prime}\right) \widetilde{\mathbb{U}}_{a}\left(t^{\prime}\right)}{\widetilde{\mathbb{U}}\left(t^{\prime}\right)},
\end{aligned}
$$

where $t^{\prime}$ and $t^{\prime}+1$ represent the time slots when the SUs observe that the PU is absent, $\widetilde{\mathbb{U}}_{a}\left(t^{\prime}\right)$ and $\widetilde{\mathbb{U}}_{\bar{a}}\left(t^{\prime}\right)$ are the average utilities of the SUs who have accessed and not accessed the channel at the $t^{\prime}$ th time slot, respectively, $\widetilde{\mathbb{U}}\left(t^{\prime}\right)$ is the average utility of all SUs when they observe that the PU is absent.

Based on the assumption that the number of SUs $M$ is sufficiently large, the portion of the SUs who sense the primary channel is equal to the probability of one individual SU choosing to sense the channel, i.e., $p_{s}=\tilde{p}_{s}$. Similarly, $p_{a}=\tilde{p}_{a}$ if the SUs observe the PU is absent. In such a case, we have

$$
\begin{aligned}
\tilde{p}_{s}(t+1) & =\frac{\tilde{p}_{s}(t) \widetilde{\mathbb{U}}_{s}(t)}{\tilde{p}_{s}(t) \widetilde{\mathbb{U}}_{s}(t)+\left(1-\tilde{p}_{s}(t)\right) \widetilde{\mathbb{U}}_{\bar{s}}(t)}, \\
& =\frac{\tilde{p}_{s}(t) \widetilde{\mathbb{U}}_{s}(t)}{\widetilde{\mathbb{U}}(t)}, \\
\tilde{p}_{a}\left(t^{\prime}+1\right) & =\frac{\tilde{p}_{a}\left(t^{\prime}\right) \widetilde{\mathbb{U}}_{a}\left(t^{\prime}\right)}{\tilde{p}_{a}\left(t^{\prime}\right) \widetilde{\mathbb{U}}_{a}\left(t^{\prime}\right)+\left(1-\tilde{p}_{a}\left(t^{\prime}\right)\right) \widetilde{\mathbb{U}}_{\bar{a}}\left(t^{\prime}\right)}, \\
& =\frac{\tilde{p}_{a}\left(t^{\prime}\right) \widetilde{\mathbb{U}}_{a}\left(t^{\prime}\right)}{\widetilde{\mathbb{U}}\left(t^{\prime}\right)} .
\end{aligned}
$$

From (79), we can see that when $\widetilde{\mathbb{U}}_{s}=\widetilde{\mathbb{U}}_{\bar{s}}$ or $\tilde{p}_{s}=0$ or $1, \dot{\tilde{p}}_{s}=$ 0 , i.e., the equilibrium is achieved. According to the replicator dynamics equation of spectrum sensing in (27), $\widetilde{\mathbb{U}}_{s}=\widetilde{\mathbb{U}}_{\bar{s}}$ and $\tilde{p}_{s}=0$ or 1 are the solutions to the replicator dynamics. The same argument can be applied to $p_{a}$. Therefore, the WrightFisher model is equivalent to the replicator dynamics equations when $M$ is sufficiently large.

From (79), we can see that in order to update $\tilde{p}_{s}(t+1)$, each SU needs to learn about the average utilities $\widetilde{\mathbb{U}}_{s}(t)$ and $\widetilde{\mathbb{U}}_{\bar{s}}(t)$. Here, we assume that each slot can be further divided into $L$ subslots and each SU uses the same strategy of channel sensing and access unchanged during all $L$ subslots. In [36], an effective utility learning method is introduced. By using the method in our system, we have (81) and (82) below, where $1 \leq n \leq L-1, \tau(n) \in(0,1)$ is a sequence of averaging factors $\sum_{n} \tau(n)=\infty$ and $\sum_{n} \tau^{2}(n)<\infty$ [36]. In this paper, we set $\tau(n)=\frac{1}{n}$. The SUs also need to learn about the average utilities $\widetilde{\mathbb{U}}_{a}(t)$ and $\widetilde{\mathbb{U}}_{\bar{a}}(t)$ to update $\tilde{p}_{a}(t+1)$ in (80). Similar to $\tilde{p}_{s}(t+1)$, we have (83) and (84) below. 


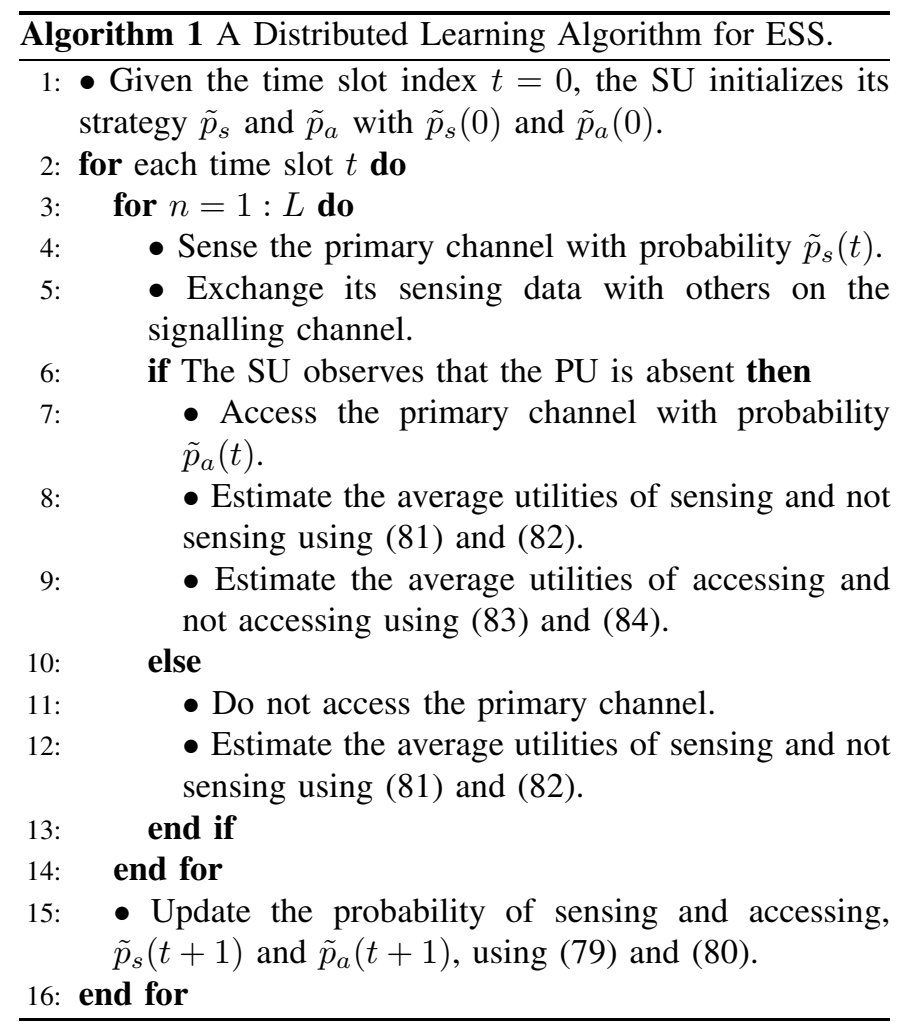

Based on (79)-(84), each SU can gradually learn the ESS. In Algorithm 1, we summarize the detailed procedures of the proposed distributed learning algorithm. In the following, we will examine the effectiveness of the learning algorithm through simulations.

\section{Simulation Results}

In this section, we conduct simulations to verify the effectiveness of our analysis. All the parameters used in the simulation are listed in Table I. We simulate the proposed learning algorithm under synchronous and asynchronous scenarios, respectively, and adjust the value of the reward $R$ to see which ESS the system will converge to.

\section{A. ESS of Synchronous and Asynchronous Scenarios}

We first illustrate the ESS of the synchronous scenario, in which all SUs use the proposed learning Algorithm 1 to update their strategies. In Fig. 5, we show the convergence of the population states $p_{s}$ and $p_{a}$, where the reward to the SUs who only contribute to sense but do not access the channel, $R$, is set as 40, 100 and 400, respectively. In the simulation, the initial population states are set as $p_{s}=0.1$ and $p_{a}=$ 0.9 , which means a large portion of SUs access the primary channel without contributing to channel sensing. From Fig. 5, we can see that with our scheme, the SUs will quickly give up such an undesired strategy, and the system finally converges to different ESSs under different settings of the reward $R$.

In Fig.5-(a), when the reward $R=40$, the ESS is $\left(p_{s}^{*}, p_{a}^{*}\right)=(0.6,0.4)$ which is corresponding to the case that part of SUs sense and access the primary channel. In such a case, $p_{s}$ converging to 0.6 is because 60 percents of SUs cooperatively sensing the channel can already achieve a relatively low false-alarm probability, and $p_{a}$ converging to 0.4 is because more than 40 percents of SUs simultaneously accessing the channel will severely impair the throughput of each other.

In Fig.5-(b), when the reward $R=100$, the ESS is $\left(p_{s}^{*}, p_{a}^{*}\right)=(1,0.25)$ which is corresponding to the case that all SUs sense but part of them access the primary channel.

$$
\begin{aligned}
& \widetilde{\mathbb{U}}_{s}(t, n+1)= \begin{cases}(1-\tau(n)) \widetilde{\mathbb{U}}_{s}(t, n)+\tau(n) U_{s a}(t, n), & \text { if the SU chooses sensing and accessing } \\
(1-\tau(n)) \widetilde{\mathbb{U}}_{s}(t, n)+\tau(n) U_{s}(t, n), & \text { if the SU chooses sensing but not accessing }\end{cases} \\
& \widetilde{\mathbb{U}}_{s}(t, n), \\
& \widetilde{\mathbb{U}}_{\bar{s}}(t, n+1)= \begin{cases}(1-\tau(n)) \widetilde{U}_{\bar{s}}(t, n)+\tau(n) U_{\bar{s} a}(t, n), & \text { if the SU chooses not sensing but accessing, } \\
(1-\tau(n)) \widetilde{U}_{\bar{s}}(t, n)+\tau(n) U_{\overline{s a}}(t, n), & \text { if the SU chooses not sensing and accessing, }\end{cases} \\
& \widetilde{\mathbb{U}}_{\bar{s}}(t, n) \text {, } \\
& \widetilde{\mathbb{U}}_{a}\left(t^{\prime}, n+1\right)= \begin{cases}(1-\tau(n)) \widetilde{U}_{a}\left(t^{\prime}, n\right)+\tau(n) U_{s a}\left(t^{\prime}, n\right), & \text { if the SU chooses sensing and accessing, } \\
(1-\tau(n)) \widetilde{\mathbb{U}}_{a}\left(t^{\prime}, n\right)+\tau(n) U_{\bar{s} a}\left(t^{\prime}, n\right), & \text { if the SU chooses not sensing but accessing, }\end{cases} \\
& \widetilde{\mathbb{U}}_{\bar{a}}\left(t^{\prime}, n+1\right)= \begin{cases}(1-\tau(n)) \widetilde{\mathbb{U}}_{\bar{a}}\left(t^{\prime}, n\right)+\tau(n) U_{s \bar{a}}\left(t^{\prime}, n\right), & \text { if the SU chooses sensing but not accessing, } \\
(1-\tau(n)) \widetilde{\mathbb{U}}_{\bar{a}}\left(t^{\prime}, n\right)+\tau(n) U_{\overline{s a}}\left(t^{\prime}, n\right), & \text { if the SU chooses not sensing and accessing, } \\
\widetilde{\mathbb{U}}_{\bar{a}}\left(t^{\prime}, n\right), & \text { if the SU chooses accessing. }\end{cases}
\end{aligned}
$$




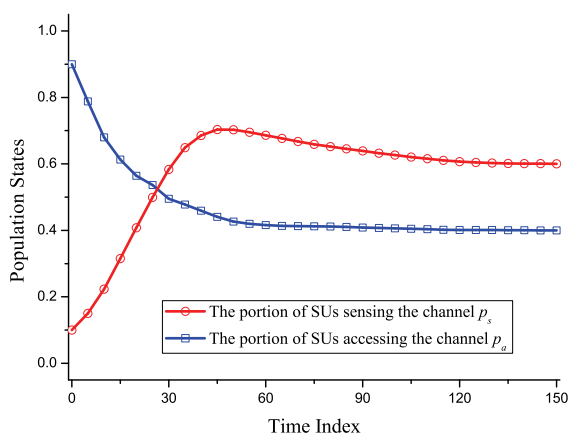

(a) $R=40$.

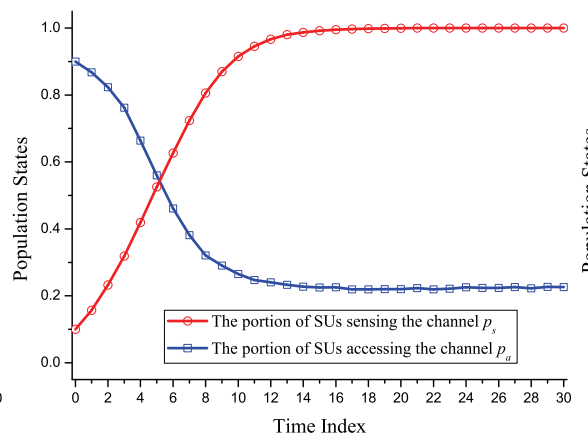

(b) $R=100$.

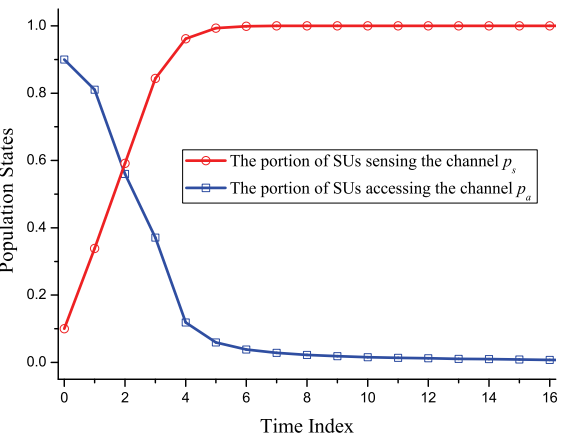

(c) $R=400$

Fig. 5. Population states of the synchronous scenario.

Although the false-alarm probability is already low enough when $p_{s}=0.6$, the increasing of the reward $R$ enhances the utility of sensing but not accessing, which attracts more SUs to sense but fewer SUs to access the primary channel.

In Fig. 5-(c), when the reward $R=400$, the ESS is $\left(p_{s}^{*}, p_{a}^{*}\right)=(1,0)$ which is corresponding to the case that all SUs sense but no one accesses the primary channel. In such a case, the reward $R$ is too high that each SU can already obtain relatively high utility from just sensing without accessing the channel. Therefore, we can see that $R$ should be properly set so that the network converges to a desired ESS.

We then illustrate the ESS of the asynchronous scenario. According to the parameters in Table I, we can calculate the SUs' access time $T_{a}=150 \mathrm{~ms}$ by (63), (64) and (70). Fig. 6 show the convergence of the population states $p_{s}$ and $p_{a}$ with different settings of the reward $R$ under the asynchronous scenario. Here, the initial population states are also set as $p_{s}=0.1$ and $p_{a}=0.9$. When the reward $R=40$ as shown in Fig. 6-(a), the ESS is $\left(p_{s}^{*}, p_{a}^{*}\right)=(0.85,0.15)$. When the reward $R=80$ as shown in Fig. 6-(b), the ESS is $\left(p_{s}^{*}, p_{a}^{*}\right)=(1,0.1)$. When the reward $R=200$ as shown in Fig. 6-(c), the ESS is $\left(p_{s}^{*}, p_{a}^{*}\right)=(1,0)$. We can see that the simulations results of these two scenarios are consistent with our ESS analysis in Section III and Section IV.

\section{B. Stability of ESS}

In order to verify the stability of the ESS, we let the SUs deviate from the equilibrium when the system is at ESS. In the synchronous scenario as shown in Fig. 7-(a), we first let the SUs deviate from cooperative sensing by setting $p_{s}=0.1$ at $t=200$ when the system has already converged to ESS. It can be seen that both $p_{s}$ and $p_{a}$ return back to the ESS quickly after the perturbation. We can also see that $p_{a}$ increases a little when $p_{s}$ falls to 0.1 . This is because a huge reduction of $p_{s}$ leads to the decrease of both $\mathbb{U}_{a}$ and $\mathbb{U}_{\bar{a}}$. If the reduction $\Delta \mathbb{U}_{a}<\Delta \mathbb{U}_{\bar{a}}, \dot{p}_{a}$ will be larger than 0 according to (8), which results in the increasing of $p_{a}$. When $t=400$, we let the SUs deviate from the equilibrium again by setting $p_{a}=0.9$. In such a case, the utility from channel access is extremely low and the SUs will not sense and access the channel. That is why $p_{s}$ begins to drop down when $p_{a}$ is set to be 0.9 . Similar phenomenons can be found in the asynchronous scenario in Fig. 7-(b).

\section{Performance Evaluation}

We first compare the performance of our distributed learning algorithm with that of the centralized algorithm. In the centralized model, there is a data center in charge of collecting each SU's utility information in each slot and globally adjusting the

TABLE I

PARAMETERS USED IN THE SIMULATION.

\begin{tabular}{c||c|c}
\hline Parameter & Value & Description \\
\hline$M$ & 20 & The number of the SUs \\
\hline$\gamma$ & $-15 \mathrm{~dB}$ & The SUs' received SNR of the PU's signal under $\mathcal{H}_{1}$ \\
\hline$P_{d}$ & 0.9 & Detection probability given by the PU \\
\hline$T_{s}$ & $10 \mathrm{~ms}$ & Sensing time of each SU \\
\hline$T_{a}$ & $100 \mathrm{~ms}$ & Accessing time of each SU \\
\hline$\lambda$ & $1 \mathrm{MHz}$ & Sampling rate of the energy detection \\
\hline$B$ & $8 \mathrm{MHz}$ & Bandwidth of the primary channel \\
\hline SNR & $-10 \mathrm{~dB}$ & Each SU's Signal-Noise-Ratio \\
\hline INR & $-20 \mathrm{~dB}$ & The Interference-Noise-Ratio from each transmitting SU \\
\hline$E_{1}$ & $0.03 \mathrm{mw} / \mathrm{bit}$ & Factor that translates one SU's throughput reward into its energy reward \\
\hline$E_{2}$ & $0.5 \mathrm{mw} / \mathrm{s}$ & Energy consumed per second by data transmission \\
\hline$E_{3}$ & $2 \mathrm{mw} / \mathrm{s}$ & Energy consumed per second by spectrum sensing \\
\hline$p_{0}$ & 0.9 & Probability that the PU is absent \\
\hline$\lambda_{0}$ & $2000 \mathrm{~ms}$ & Average length of the OFF state \\
\hline$\lambda_{1}$ & $222 \mathrm{~ms}$ & Average length of the ON state \\
\hline$R_{a v}^{\downarrow}$ & $10 \mathrm{Mbps}$ & PU's minimum average data rate \\
\hline$R_{p}$ & $15 \mathrm{Mbps}$ & PU's data rate when there is no interference from the SUs \\
\hline & &
\end{tabular}




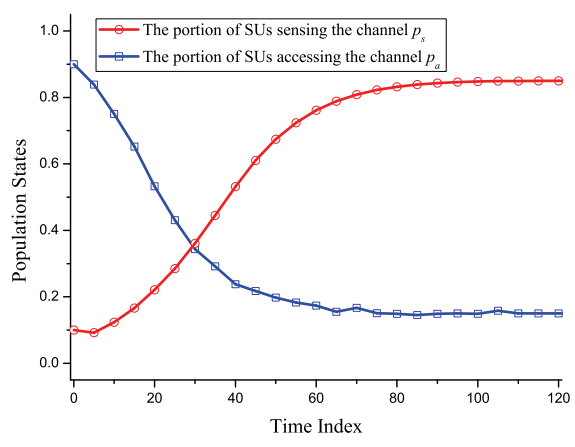

(a) $R=40$.

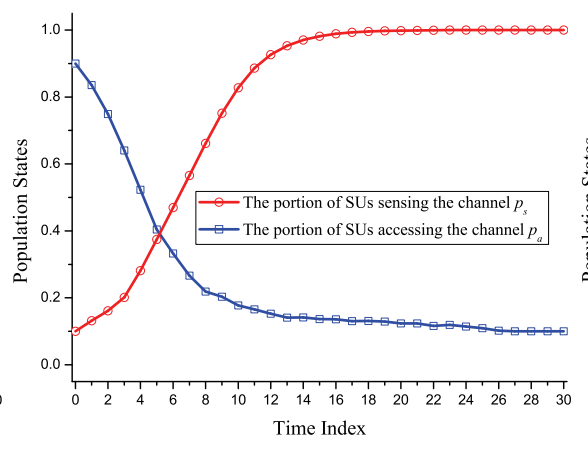

(b) $R=80$.

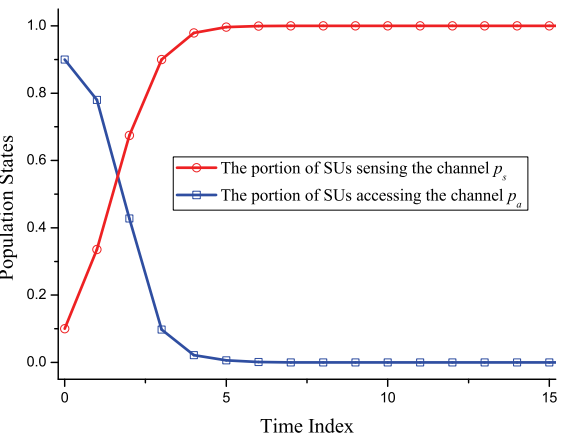

(c) $R=200$.

Fig. 6. Population states of the asynchronous scenario.

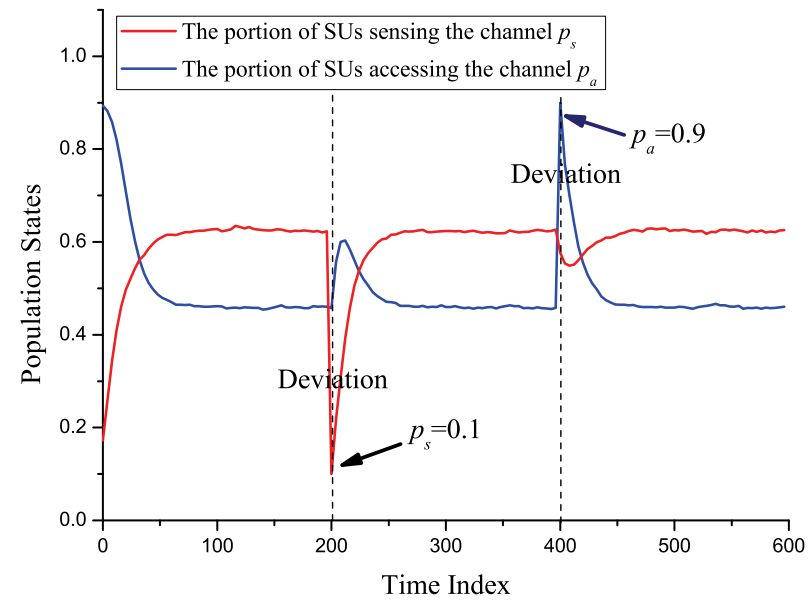

(a) Synchronous scenario with $R=50$.

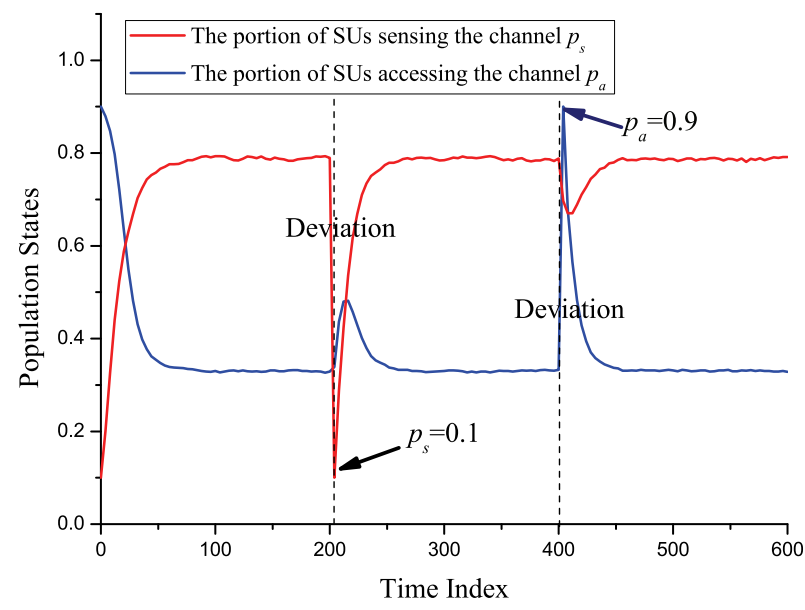

(b) Asynchronous scenario with $R=30$.

Fig. 7. Stability of ESS.

SUs' strategies $p_{s}$ and $p_{a}$ in the next time slot. Fig. 8 shows the comparison results in terms of the average utility of all SUs, from which we can see that the gap between our distributed algorithm and the centralized one is about 6\%. Nevertheless, the centralized algorithm requires all the SUs to truthfully report their private utility information, while our distributed algorithm does not.

We further conduct simulation to evaluate the performance of our joint channel sensing and access algorithm. Fig. 9 shows the performances of SUs' false-alarm probability and

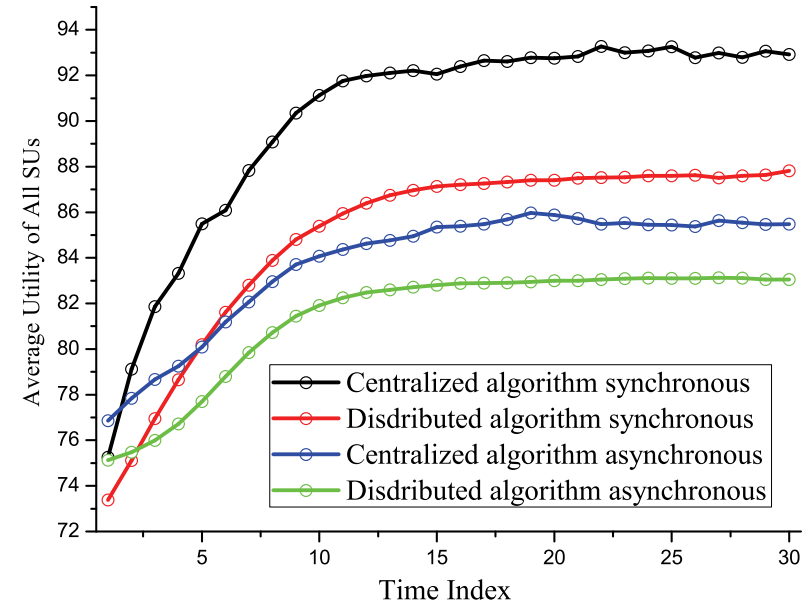

Fig. 8. Utility comparison under synchronous scenario with $R=50$ and asynchronous scenario with $R=30$.

throughput during the ESS convergence process under synchronous and asynchronous scenarios, respectively. We can see that along with the system converging to the ESS for both scenarios, the false-alarm probability gradually tends to the lowest limit, while the throughput gradually achieve the highest limit. Moreover, from Fig. 5-(b), Fig. 6-(b) and Fig. 9, we can see that the false-alarm probability is a decreasing function in terms of $p_{s}$ and the SUs' throughput is a decreasing function in terms of $p_{a}$, which is consistent with the results proved in Lemma 1.

\section{CONCLUSION}

In this paper, we analyzed how the SUs should cooperate with each other in the joint spectrum sensing and access problem using evolutionary game theory. We studied the behavior dynamics of the SUs under two scenarios: synchronous and asynchronous scenarios. Through solving the joint replicator dynamics equations of channel sensing and accessing, we derived different ESSs under different conditions. Based on the nature selection theory, we proposed a distributed learning algorithm that enable the SUs to achieve the ESSs purely based on their own utility histories. From simulation results, we can see that by adjusting the reward to the contributors, the population states of the network will converge to the desired ESS. 


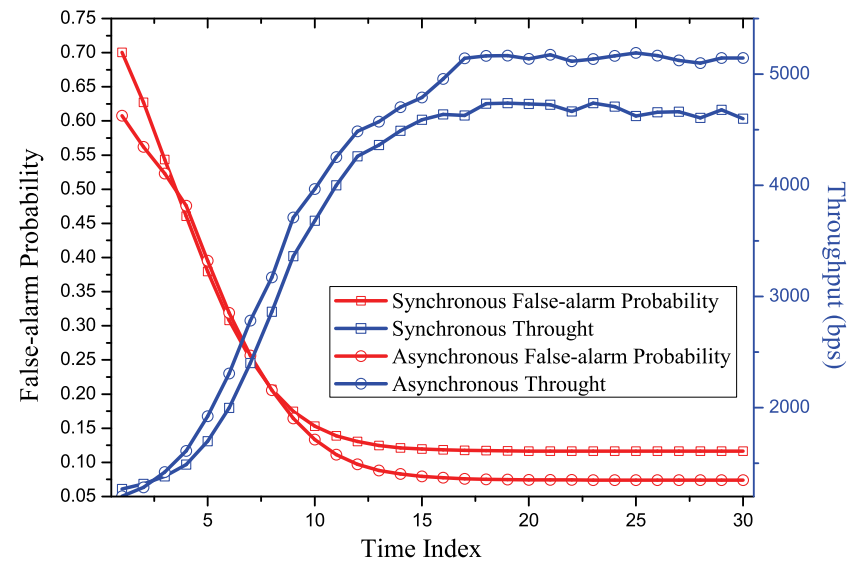

Fig. 9. Sensing and access performances under synchronous scenario with $R=100$ and asynchronous scenario with $R=80$.

The system model discussed in this paper is single-channel case. An extension of this work is to study the multi-channel case. In the multi-channel case, we can use Markov process to model the channel state transition process, where the system state space is the set of all the possible combinations of all channels' states (if we assume the channels are independent). In such a case, the utility functions are dependent on the system state, while the analysis of replicator dynamics and ESS derivations are similar to the single-channel case. Since the system state space will increase exponentially with the number of channels, a fast suboptimal learning algorithm should be designed for the SUs to converge the ESS.

\section{REFERENCES}

[1] S. Haykin, "Cognitive radio: brain-empowered wireless communications," IEEE J. Sel. Areas Commun., vol. 23, no. 2, pp. 201-220, 2005.

[2] B. Wang and K. J. R. Liu, "Advances in cognitive radios: a survey," IEEE J. Sel. Topics Signal Process., vol. 5, no. 1, pp. 5-23, 2011.

[3] K. J. R. Liu and B. Wang, Cognitive Radio Networking and Security: A Game Theoretical View. Cambridge University Press, 2010.

[4] S. Shankar, C. Cordeiro, and K. Challapali, "Spectrum agile radios: utilization and sensing architectures," in Proc. 2005 IEEE DySPAN, pp. $160-169$.

[5] H. Tang, "Some physical layer issues of wide-band cognitive radio systems," in Proc. 2005 IEEE DySPAN, pp. 151-159.

[6] A. Ghasemi and E. S. Sousa, "Collaborative spectrum sensing for opportunistic access in fading environments," in Proc. 2005 IEEE DySPAN, pp. 131-136.

[7] E. Visotsky, S. Kuffner, and R. Peterson, "On collaborative detection of TV transmissions in support of dynamic spectrum sharing," in Proc. 2005 IEEE DySPAN, pp. 338-345.

[8] Y.-C. Liang, Y. Zeng, E. C. Peh, and A. T. Hoang, "Sensing-throughput tradeoff for cognitive radio networks," IEEE Trans. Wireless Commun., vol. 7, no. 4, pp. 1326-1337, 2008.

[9] R. Fan and H. Jiang, "Optimal multi-channel cooperative sensing in cognitive radio networks," IEEE Trans. Wireless Commun., vol. 9, no. 3, pp. 1128-1138, 2010.

[10] F. Zeng, C. Li, and Z. Tian, "Distributed compressive spectrum sensing in cooperative multihop cognitive networks," IEEE J. Sel. Topics Signal Process., vol. 5, no. 1, pp. 37-48, 2011.

[11] Q. Zhao, L. Tong, A. Swami, and Y. Chen, "Decentralized cognitive MAC for opportunistic spectrum access in Ad Hoc networks: a POMDP framework," IEEE J. Sel. Areas Commun., vol. 25, no. 3, pp. 589-600, 2007.

[12] A. K. Sadek, K. J. R. Liu, and A. Ephremides, "Cognitive multiple access via cooperation: protocol design and stability analysis," IEEE Trans. Inf. Theory, vol. 53, no. 10, pp. 3677-3696, 2007.

[13] Z. Ji and K. J. R. Liu, "Dynamic spectrum sharing: a game theoretical overview," IEEE Commun. Mag., vol. 45, no. 5, pp. 88-94, 2010.
[14] J. Unnikrishnan and V. V. Veeravalli, "Algorithms for dynamic spectrum access with learning for cognitive radio," IEEE Trans. Signal Process., vol. 58 , no. 2, pp. 750-760, 2010.

[15] A. A. El-Sherif, A. Kwasinski, A. Sadek, and K. J. R. Liu, "Contentaware cognitive multiple access protocol for cooperative packet speech communications," IEEE Trans. Wireless Commun., vol. 8, no. 2, pp. 995-1005, 2009.

[16] A. A. El-Sherif, A. K. Sadek, and K. J. R. Liu, "Opportunistic multiple access for cognitive radio networks," IEEE J. Sel. Areas Commun., vol. 29, no. 4, pp. 704-715, 2011.

[17] Y. Wu, B. Wang, K. J. R. Liu, and T. C. Clancy, "A scalable collusionresistant multi-winner cognitive spectrum auction game," IEEE Trans. Wireless Commun., vol. 57, no. 12, pp. 3805-3816, 2009.

[18] D. Li, Y. Xu, X. Wang, and M. Guizani, "Coalitional game theoretic approach for secondary spectrum access in cooperative cognitive radio networks," IEEE Trans. Wireless Commun., vol. 10, no. 3, pp. 844-856, 2011.

[19] A. A. El-Sherif and K. J. R. Liu, "Joint design of spectrum sensing and channel access in cognitive radio networks," IEEE Trans. Wireless Commun., vol. 10, no. 6, pp. 1743-1753, 2011.

[20] W. Saad, Z. Han, R. Zheng, A. Hjorungnes, T. Basar, and H. V. Poor, "Coalitional games in partition form for joint spectrum sensing and access in cognitive radio networks," IEEE J. Sel. Topics Signal Process., vol. 6, no. 2, pp. 195-209, 2012.

[21] Y. Chen, Y. Gao, and K. J. R. Liu, "An evolutionary game-theoretic approach for image interpolation," in Proc. 2011 IEEE ICASSP, pp. 989-992.

[22] R. Cressman, Evolutionary Dynamics and Extensive Form Games. MIT Press, 2003.

[23] B. Wang, Y. Wu, and K. J. R. Liu, "Game theory for cognitive radio networks: an overview," Computer Networks, vol. 54, no. 14, pp. 2537 2561, 2010.

[24] E. H. Watanabe, D. Menasché, E. Silva, and R. M. Leão, "Modeling resource sharing dynamics of VoIP users over a WLAN using a gametheoretic approach," in Proc. 2008 IEEE INFOCOM, pp. 915-923.

[25] B. Wang, K. J. R. Liu, and T. C. Clancy, "Evolutionary cooperative spectrum sensing game: how to collaborate?" IEEE Trans. Commun., vol. 58, no. 3, pp. 890-900, 2010.

[26] Y. Chen, B. Wang, W. S. Lin, Y. Wu, and K. J. R. Liu, "Cooperative peer-to-peer streaming: an evolutionary game-theoretic approach," IEEE Trans. Circuit Syst. Video Technol., vol. 20, no. 10, pp. 1346-1357, 2010.

[27] P. Baronti, P. Pillai, V. Chook, S. Chessa, A. Gotta, and Y. Hu, "Wireless sensor networks: a survey on the state of the art and the 802.15.4 and zigbee standards," Computer Commun., vol. 30, no. 7, pp. 1655-1695, 2007.

[28] C. Buratti, A. Conti, D. Dardari, and R. Verdone, "An overview on wireless sensor networks technology and evolution," Sensors, vol. 9, no. 9, pp. 6869-6896, 2009.

[29] I. F. Akyildiz, B. F. Lo, and R. Balakrishnan, "Cooperative spectrum sensing in cognitive radio networks: a survey," Physical Commun., vol. 4, no. 3, pp. 40-62, 2011.

[30] C. Jiang, Y. Chen, K. J. R. Liu, and Y. Ren, "Renewal-theoretical dynamic spectrum access in cognitive radio networks with unknown primary behavior," IEEE J. Sel. Areas Commun., vol. 31, no. 3, pp. $1-11,2013$.

[31] C. Jiang, Y. Chen, and K. J. R. Liu, "A renewal-theoretical framework for dynamic spectrum access with unknown primary behavior," in Proc. 2012 IEEE Globecom, pp. 1-6.

[32] C. Jiang, Y. Chen, K. J. R. Liu, and Y. Ren, "Analysis of interference in cognitive radio networks with unknown primary behavior," in Proc. 2012 IEEE ICC, pp. 1746-1750.

[33] H. Kim and K. G. Shin, "Efficient discovery of spectrum opportunities with MAC-layer sensing in cognitive radio networks," IEEE Trans. Mobile Computing, vol. 7, no. 5, pp. 533-545, 2008.

[34] D. R. Cox, Renewal Theory. Butler and Tanner, 1967.

[35] R. Fisher, The Genetical Theory of Natural Selection. Clarendon Press, 1930.

[36] R. Cominetti, E. Melo, and S. Sorin, "A payoff-based learning procedure and its application to traffic games," Games and Economic Behavior, vol. 70, no. 1, pp. 71-83, 2010. 


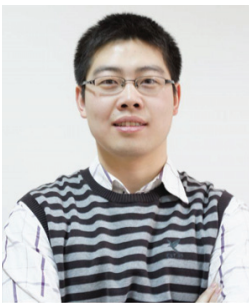

Chunxiao Jiang (S'09-M'13) received his B.S. degree in information engineering from Beijing University of Aeronautics and Astronautics (Beihang University) in 2008 and the Ph.D. degree from Tsinghua University (THU), Beijing in 2013, both with the highest honors. During 2011-2012, he visited the Signals and Information Group (SIG) at Department of Electrical \& Computer Engineering (ECE) of University of Maryland (UMD), supported by China Scholarship Council (CSC) for one year.

Dr. Jiang is currently a research associate in ECE department of UMD with Prof. K. J. Ray Liu, and also a post-doctor in EE department of THU. His research interests include the applications of game theory and queuing theory in wireless communication and networking and social networks.

Dr. Jiang received the Beijing Distinguished Graduated Student Award in 2013, Chinese National Fellowship in 2012, Outstanding Scholarship in Tsinghua University in 2009, and was candidate of Microsoft Research Asia Fellowship in 2010.

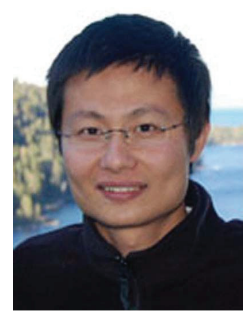

Yan Chen (S'06-M'11) received the Bachelor's degree from University of Science and Technology of China in 2004, the M. Phil degree from Hong Kong University of Science and Technology (HKUST) in 2007, and the Ph.D. degree from University of Maryland College Park in 2011.

$\mathrm{He}$ is currently a research associate in the Department of Electrical and Computer Engineering at University of Maryland College Park. His current research interests are in social learning and networking, smart grid, cloud computing, crowdsourcing, network economics, multimedia signal processing and communication.

Dr. Chen received the University of Maryland Future Faculty Fellowship in 2010, Chinese Government Award for outstanding students abroad in 2011, University of Maryland ECE Distinguished Dissertation Fellowship Honorable Mention in 2011, and was the Finalist of A. James Clark School of Engineering Dean's Doctoral Research Award in 2011.

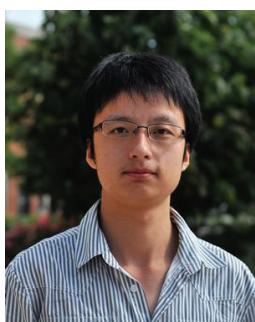

Yang Gao (S'12) received the B.S. in Electronic Engineering from Tsinghua University, Beijing, China in 2009. Now he is a Ph.D. student in the Department of Electrical and Computer Engineering at University of Maryland, College Park. His current research interests are in the area of network economics, crowdsourcing and smart grid. He received the silver medal of the 21 st National Chinese Physics Olympiad, the national scholarship from the Ministry of Education of People's Republic of China in 2008, the honor of Outstanding Graduate of Tsinghua University in 2009 and the University of Maryland Future Faculty Fellowship in 2012.

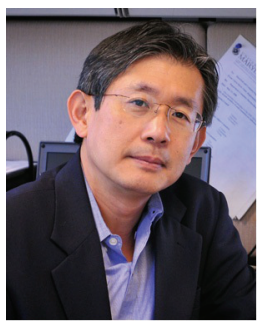

K. J. Ray Liu (F'03) was named a Distinguished Scholar-Teacher of University of Maryland, College Park, in 2007, where he is Christine Kim Eminent Professor of Information Technology. He leads the Maryland Signals and Information Group conducting research encompassing broad areas of signal processing and communications with recent focus on cooperative communications, cognitive networking, social learning and networks, and information forensics and security.

Dr. Liu is the recipient of numerous honors and awards including IEEE Signal Processing Society Technical Achievement Award and Distinguished Lecturer. He also received various teaching and research recognitions from University of Maryland including university-level Invention of the Year Award; and Poole and Kent Senior Faculty Teaching Award and Outstanding Faculty Research Award, both from A. James Clark School of Engineering. An ISI Highly Cited Author, Dr. Liu is a Fellow of IEEE and AAAS.

Dr. Liu is President of IEEE Signal Processing Society where he has served as Vice President - Publications and Board of Governor. He was the Editor-inChief of IEEE Signal Processing Magazine and the founding Editor-in-Chief of EURASIP Journal on Advances in Signal Processing. 